\title{
CK2 $\alpha /$ CSNK2A1 Phosphorylates SIRT6 and Is Involved in the Progression of Breast Carcinoma and Predicts Shorter Survival of Diagnosed Patients
}

Jun Sang Bae, ${ }^{*}$ See-Hyoung Park, ${ }^{\dagger}$ Urangoo Jamiyandorj, ${ }^{*}$ Kyoung Min Kim,${ }^{*}$ Sang Jae Noh,,${ }^{\ddagger}$ Jung Ryul Kim, Hye Jeong Park, ${ }^{*}$ Keun Sang Kwon, "Sung Hoo Jung, Ho Sung Park, ${ }^{*}$ Byung-Hyun Park, ** Ho Lee, ${ }^{\ddagger}$ Woo Sung Moon, * Karl G. Sylvester, ${ }^{\dagger \dagger}$ and Kyu Yun Jang*

\begin{abstract}
From the Departments of Pathology, * Forensic Medicine, ${ }^{\ddagger}$ Orthopedic Surgery, ${ }^{\S}$ Preventive Medicine, ${ }^{\llbracket}$ Surgery,, and Biochemistry, ** Chonbuk National University Medical School, Research Institute of Clinical Medicine of Chonbuk National University-Biomedical Research Institute of Chonbuk National University Hospital and Research Institute for Endocrine Sciences, Jeonju, Republic of Korea; the Department of Bio and Chemical Engineering, ${ }^{\dagger}$ Hongik University, Sejong, Republic of Korea; and the Department of Surgery, ${ }^{\dagger \dagger}$ Division of Pediatric Surgery, Stanford University School of Medicine, Stanford, California
\end{abstract}

\author{
Accepted for publication \\ August 10, 2016. \\ Address correspondence to \\ Kyu Yun Jang, M.D., Ph.D., \\ Department of Pathology, \\ Chonbuk National University \\ Medical School, 567 \\ Baekje-daero, Dukjin-gu, \\ Jeonju 561-756, Republic of \\ Korea. E-mail: kyjang@ \\ chonbuk.ac.kr.
}

\begin{abstract}
Recently, the roles of sirtuins (SIRTs) in tumorigenesis have been of interest to oncologists, and protein kinase CK2 $\alpha 1$ (CSNK2A1) has been shown to be involved in tumorigenesis by phosphorylating various proteins, including SIRT1. Therefore, we evaluated the roles of CSNK2A1, SIRT6, and phosphorylated SIRT6 and their relationships in breast carcinoma. Nuclear expression of CSNK2A1 and SIRT6 predicted shorter overall survival and relapse-free survival by multivariate analysis. Inhibition of CSNK2A1 decreased the proliferative and invasive activity of cancer cells. In addition, CSNK2A1 was bound to SIRT6 and phosphorylated SIRT6; evidence for this is provided from immunofluorescence staining, co-immunoprecipitation of CSNK2A1 and SIRT6, a glutathione S-transferase pull-down assay, an in vitro kinase assay, and transfection of mutant CSNK2A1. Knockdown of SIRT6 decreased the proliferation and invasiveness of cancer cells. Overexpression of SIRT6 increased proliferation, but mutation at the Ser338 phosphorylation site of SIRT6 inhibited the proliferation of MCF7 cells. Moreover, both knockdown of SIRT6 and a mutation at the phosphorylation site of SIRT6 decreased expression of matrix metallopeptidase 9, $\beta$-catenin, cyclin D1, and NF- $\kappa$ B. Especially, SIRT6 expression was associated with the nuclear localization of $\beta$-catenin. This study demonstrates that CSNK2A1 and SIRT6 are indicators of poor prognosis for breast carcinomas and that CSNK2A1-mediated phosphorylation of SIRT6 might be involved in the progression of breast carcinoma. (Am J Pathol 2016, 186: 3297-3315; http:// dx.doi.org/10.1016/j.ajpath.2016.08.007)
\end{abstract}

Protein kinase CK2 (designated CSNK2) is a highly conserved serine/threonine kinase involved in various aspects of cell biology and $>300$ targets of CSNK2 are known. ${ }^{1}$ CSNK2 phosphorylates various proteins related with regulation of cell cycle, survival of cells, cell morphology, metabolism, tumorigenesis, and invasiveness of cancer cells. ${ }^{1-3}$ Because the expression and kinase activity of casein CSNK2 $\alpha 1$ (CSNK2A1) was higher in malignant tumor cells than normal counterpart cells in stomach, ${ }^{4}$ kidney, ${ }^{5}$ colorectum, ${ }^{6}$ and prostate, ${ }^{7}$ it has been suggested that CSNK2A1 has a tumorigenic role. In mammary glands, overexpression of CSNK2A1 correlated with increased tumorigenesis in transgenic mice ${ }^{8}$ and with distant metastasis in breast carcinoma (BCA) patients. ${ }^{9}$ The oncogenic role of CSNK2 was further supported by its

\footnotetext{
Supported by the National Research Foundation of Korea (NRF) funded by the Korean government (MSIP) grant no. 2008-0062279. The biospecimens for this study were provided by the Biobank of Chonbuk National University Hospital, a member of the National Biobank of Korea, which is supported by the Ministry of Health, Welfare and Family Affairs. All samples derived from the National Biobank of Korea were obtained with informed consent under institutional review board-approved protocols.

J.S.B., S.-H.P., and U.J. contributed equally to this work.

Disclosures: None declared.
} 
activation of signals related with tumor progression, such as c-Myc, ${ }^{8,10} \mathrm{Wnt} / \beta$-catenin pathway, ${ }^{8,11} \mathrm{NF}-\kappa \mathrm{B},{ }^{12}$ sirtuin 1 (SIRT1), ${ }^{13}$ and epithelial-mesenchymal transition. ${ }^{4,6}$ In addition, CSNK2A1 inhibited tumor suppressors, such as phosphatase and tensin homolog ${ }^{14}$ and p53. ${ }^{15}$ Especially, the antiapoptotic function of CSNK2 was closely associated with resistance to chemotherapeutic agents, ${ }^{1-3,16}$ Moreover, higher expression of CSNK2A1 in cancers is associated with poor survival of patients with colon cancer, ${ }^{6,17}$ gastric cancer, ${ }^{4}$ and prostate cancer. ${ }^{7}$ Therefore, CSNK2 could be a potential therapeutic target of human malignant tumors.

There are seven types of SIRTs (SIRT1 to SIRT7) with diverse roles in aging, metabolism, and tumorigenesis according to the type of SIRTs. ${ }^{18-24}$ Among them, research on SIRT6 has focused on its beneficial role in aging ${ }^{23}$ and metabolism. ${ }^{22}$ However, the reports regarding a role of SIRT6 in tumorigenesis are controversial. SIRT6 has been suggested as a tumor suppressor in $\operatorname{liver}^{25}$ and breast ${ }^{26}$; however, other reports have shown SIRT6 to have potential as a tumorigenic molecule in $\mathrm{BCA}^{21}$ skin cancer, ${ }^{27}$ pancreatic cancer, $^{28}$ and hepatocellular carcinoma. $^{29}$ Therefore, a careful approach to the controlling of SIRT6 as a therapeutic target of cancer patients and further study to clarify the role of SIRT6 in cancers is needed.

CSNK2 has a diverse role as a protein kinase and is involved in the regulation of the function of SIRTs. Especially, CSNK2A1 phosphorylates SIRT1 and regulates the substrate-binding affinity of SIRT1. ${ }^{13,30}$ Therefore, there is a possibility that CSNK2A1 is also involved in the phosphorylation of other types of SIRTs. In addition, investigating of the relationship between CSNK2A1 and SIRT6 could be helpful in understanding the role of SIRT6 in tumorigenesis. From this rationale, we investigated the role of CSNK2, especially the catalytic subunit CSNK2A1, in the functional regulation of SIRT6 in BCA cells. In particular, the phosphorylation of SIRT6 on Ser338 among the four phosphorylation sites has been known to be important in the function of SIRT6. ${ }^{26,31}$ Therefore, we focused on whether Ser338 SIRT6 phosphorylation is affected by CSNK2A1. In addition, we investigated the relationship between the expression of CSNK2A1, SIRT6, and phosphorylated SIRT6 (pSIRT6) in human BCA tissue samples and their prognostic impact on human BCA patients.

\section{Materials and Methods}

\section{Patients and Tissue Samples}

BCA patients diagnosed in Chonbuk National University Hospital between January 1997 and August 2002 were subjected to this study. One hundred forty-two female BCA cases with available medical records, histologic slides, and paraffin-embedded tissue blocks were included in this study. The age of the 142 BCA patients ranged from 22 to 72 years (mean, 46.7 years). Eighty-seven patients received modified radical mastectomy, and 55 patients received breast conserving surgery. One hundred twenty-six patients received systemic adjuvant chemotherapy (cyclophosphamide, methotrexate, and fluorouracil chemotherapy or anthracycline- and taxane-based chemotherapy) and 119 patients received adjuvant endocrine therapy. One hundred eight patients received both chemotherapy and endocrine therapy, and 5 patients received no adjuvant therapy. The median follow-up duration was 148.8 months (range, 7.7 to 198.6). Among the 142 BCA patients, 34 patients experienced relapse and 44 patients died of BCA at the follow-up end point. The 5- and 10-year survival rates for all of the BCA patients were $81 \%$ and $74 \%$, respectively. All of the cases were reviewed and classified by two pathologists (K.Y.J. and S.J.N.), each with more than 5 years' experience in the Department of Pathology) according to the World Health Organization Classification. ${ }^{32}$ Tumor stage was assigned according to the seventh edition of the American Joint Committee on Cancer staging system. ${ }^{33}$ The histologic diagnoses of 142 cases of BCA were 134 invasive carcinomas of no special type and 8 invasive lobular carcinomas. The BCA patients were grouped according to their age $[<50$ years $(n=101)$ versus $\geq 50$ years $(n=41)$ ], TNM stage [I $(n=26)$ versus II $(n=97)$ versus III and IV $(n=19)]$, T stage $[1(n=38)$ versus $2(n=95)$ versus 3 and 4 $(n=9)$ ], lymph node metastasis [absence $(n=80)$ versus presence $(n=62)$ ], distant metastatic relapse [absence $(n=107)$ versus presence $(n=35)]$, histologic type [invasive carcinoma of no special type $(n=134)$ versus invasive lobular carcinoma $(n=8)$ ], mitotic count [0 to 9 $(n=91)$ versus 10 to $19(n=26)$ versus $>19 / 10$ highpower field $(n=25)]$, histologic grade $[1(n=49)$ versus $2(n=64)$ versus $3(n=29)]$, and immunohistochemical expression of human epidermal growth factor receptor 2 (HER2) [negative $(n=98)$ versus positive $(n=44)$ ], estrogen receptor (ER) [negative $(n=63)$ versus positive $(n=79)$ ], and progesterone receptor $(\mathrm{PR})$ [negative $(n=59)$ versus positive $(n=83)]$. This study obtained institutional review board approval from Chonbuk National University Hospital (CUH 2014-03-002-001), and written informed consents were obtained from the patients or their legal guardian. All experiments were performed in accordance with relevant guidelines and regulations.

Immunohistochemical Staining and Immunohistochemical Scoring for Tissue Microarrays

To establish tissue-microarray blocks, two 3.0-mm sized tissue cores per case were obtained from the most representative area composed mainly of tumor cells. Antigen retrieval for the tissue sections were performed with a microwave oven in Dako Target Retrieval Solution (pH 6.0; Dako, Glostrup, Denmark) for 12 minutes. Thereafter, immunohistochemical staining for CSNK2A1 (dilution 1:100; polyclonal; Cell Signaling Technology, Beverly, MA), SIRT6 (dilution 1:50; polyclonal; Lifespan Biosciences, Seattle, WA), and pSIRT6 (Ser338) 
(dilution 1:100; polyclonal, Bioss Antibodies, Atlanta, GA) was performed. The expression of CSNK2A1 was evaluated in the nuclei of tumor cells because nuclear expression of CSNK2A1 was shown to be related to the prognosis of human cancers. ${ }^{4,6}$ Because the prognostic implication of SIRT6 expression was different according to the localization of SIRT6 expression, immunostaining for SIRT6 and pSIRT6 was separately evaluated for cytoplasmic or nuclear expression. ${ }^{21}$ The scoring for the immunohistochemical-stained slides was performed by two pathologists (K.Y.J. and S.J.N.) by consensus without knowledge of the clinicopathologic information. Immunohistochemical staining of CSNK2A1, SIRT6, and pSIRT6 was evaluated by the sum of the staining intensity scores and the staining area scores in each tissue-microarray core. The staining intensity was scored as 0 (no staining), 1 (weak staining), 2 (intermediate staining), and 3 (strong staining). The staining area was scored as 0 (no stained cells), 1 ( $1 \%$ of the cells stained positive), 2 ( $2 \%$ to $10 \%$ of the cells stained positive), 3 ( $11 \%$ to $33 \%$ of the cells stained positive), 4 (34\% to $66 \%$ of the cells stained positive), and 5 (67\% to $100 \%$ of the cells stained positive). ${ }^{34,35}$ Thereafter, the combined score obtained by adding the sum of the scores of two different tissue-microarray cores and used for further analysis. ${ }^{35-37}$ The combined score ranged from 0 to 16 . Subsequently, the expression of CSNK2A1, nuclear SIRT6, cytoplasmic SIRT6, nuclear pSIRT6, or cytoplasmic pSIRT6 were grouped as positive or negative by receiver operating characteristic curve analysis at the highest positive likelihood ratio point for the death of BCA patients. HER2 expression was considered positive if $\geq 10 \%$ of the tumor cells showed complete and intense membranous staining. The expression of ER and PR was considered positive if $\geq 1 \%$ of the tumor cells showed nuclear staining.

\section{Cell Culture and Chemicals}

Human breast cancer cell lines, MCF7, T47D, MDA-MB231, and 293 cells were purchased from the Korean Cell Line Bank (Seoul, Korea). The cell lines were maintained in RPMI 1640, Dulbecco's modified Eagle's medium (DMEM), and minimal essential medium supplemented with $10 \%$ fetal bovine serum (FBS) and penicillin/streptomycin (100 U/mL) (Gibco BRL, Gaithersburg, MD). Two CSNK2 inhibitors, CX4945 (Santa Cruz Biotechnology, Santa Cruz, CA) and emodin (Sigma-Aldrich, St. Louis, MO), were used. The $\mathrm{EC}_{50}$ of CX4945 in T47D and MCF7 breast cancer cell lines were reported as $8.39 \mu \mathrm{mol} / \mathrm{L}$ and $8.88 \mu \mathrm{mol} / \mathrm{L}$, respectively. ${ }^{38}$ Therefore, we used $2,6,8$, and $10 \mu \mathrm{mol} / \mathrm{L}$ CX4945 in this study. Emodin was effective at inhibiting the proliferation and induction of apoptosis in human cancer cells at concentrations between 20 and $60 \mu \mathrm{mol} / \mathrm{L}$. ${ }^{4,39,40}$ Therefore, we used 20,30 , and $40 \mu \mathrm{mol} / \mathrm{L}$ emodin in this study.
Transfection of siRNAs, shRNAs, and Plasmids

The siRNAs for CSNK2A1 and SIRT6 were synthesized by Bioneer Corporation (Daejeon, Korea). The siRNA target sense and antisense sequences are as follows: CSNK2A1, 5'-CAUUUAGUUACUGGGCAUA- $3^{\prime}$ and 5'-UAUGCCCAGUAACUAAAUG-3'; SIRT6, 5'-CAGCUAAACAGGAGUGAA- $3^{\prime}$ and $5^{\prime}$-UUCACUCCUGUUUAAGCUG- $3^{\prime}$; negative control, $5^{\prime}$-CCUACGCCACCAAUUUCGU- $3^{\prime}$ and $5^{\prime}$-ACGAAAUUGGUGGCGUAGG-3'. The CSNK2A1- or SIRT6-specific shRNA expression vectors were purchased from GenePharma Co. (Shanghai, China). The CSNK2A1 duplex had the sense and antisense sequences $5^{\prime}$ CACCGGGTGAAACACTTCAGAAGCATTCAAGAGATGCTTCTGAAGTGTTTCACCCTTTTTTG- $3^{\prime}$ and $5^{\prime}$ GATCCAAAAAAGGGTGAAACACTTCAGAAGCATCTCTTGAATGCTTCTGAAGTGTTTCACCC- $3^{\prime}$, respectively. The SIRT6 duplex had the sense and antisense sequences 5'-CACCGCTACGTTGACGAGGTCATGATTCAAGAGATCATGACCTCGTCAACGTAGCTTTTTTG$3^{\prime}$ and 5'-GATCCAAAAAAGCTACGTTGACGAGGTCATGATCTCTTGAATCATGACCTCGTCAACGTAGC$3^{\prime}$, respectively. A pFLAG-CMV-2 plasmid vector was used as control vector. The plasmids for HA-tagged wild-type (WT) CSNK2Al (pRc/CMV_CSNK2A1-HA) and for HAtagged mutant CSNK2A1 (pRc/CMV_CSNK2A1_K68MHA) were kindly provided by Laszlo Gyenis (Department of Biochemistry, University of Western Ontario, Canada). The plasmids for WT SIRT6 (pFLAG2_SIRT6) and mutant construct for the Thr294 (pFLAG2_SIRT6_T294A), Ser303 (pFLAG2_SIRT6_S303A), Ser330 (pFLAG2_SIRT6_S330A), and Ser338 (pFLAG2_SIRT6_S338A) phosphorylation sites of SIRT6 were synthesized by Cosmo Genetech Co. Ltd. (Seoul, Korea). The $\beta$-catenin-specific shRNA expression vector was purchased from Santa Cruz Biotechnology (no. sc-29209-SH). Lipofectamine RNAiMAX or Lipofectamine 2000 (Invitrogen, Carlsbad, CA) was used for transfection.

\section{Cell Proliferation and Cell Counting Assay}

The proliferation of cells was evaluated with a MTT cell proliferation assay (Sigma-Aldrich) and a colony-forming assay. For the MTT assay, $5 \times 10^{3}$ MCF7, $5 \times 10^{3}$ T47D, or $3 \times 10^{3}$ MDA-MB-231 cells were seeded, and the absorbance was measured at $560 \mathrm{~nm}$. For the cell counting assay, $1 \times 10^{3}$ cells were seeded in 6 -cm culture plates and incubated for $0,24,72$, and 90 hours. For the colonyforming assay, $5 \times 10^{3}$ cells were cultured in 24-well culture plates for 10 days.

\section{Soft Agar Assay}

For the soft agar colony formation assay, the bottom of a $35-\mathrm{mm}$ dish was coated with $1 \mathrm{~mL}$ of RPMI 1640 with $10 \%$ FBS and $1 \%$ low-melting agarose and covered in $1 \mathrm{~mL}$ of 
RPMI 1640 with $10 \%$ FBS and $0.5 \%$ agarose containing $5 \times 10^{3}$ cells. The cells were maintained at $37^{\circ} \mathrm{C}$ in a humidified $5 \% \mathrm{CO}_{2}$ atmosphere for 2 weeks. Then, the number colony was counted in five microscopic fields (magnification, $\times 100$ ).

\section{Western Blot Analysis and Subcellular Protein Fractionation}

Total proteins were lysed with PRO-PREP Protein Extraction Solution (iNtRON Biotechnology Inc., Seongnam, Korea) containing $1 \times$ phosphatase inhibitor cocktails 2, 3 (Sigma-Aldrich) and probed with primary antibodies for CSNK2A1 (polyclonal; Cell Signaling Technology), SIRT6 (clone D8D12; Cell Signaling Technology), pSIRT6 (Ser338) (polyclonal; Bioss Antibodies), SIRT1 (clone H-300; Santa Cruz Biotechnology), pSIRT1 (Ser47) (polyclonal; Cell Signaling Technology), p53 (clone DO-7; Novocastra, Newcastle, United Kingdom), acetylated p53 (Lys382) (polyclonal; Cell Signaling Technology), forkhead box O3 (FOXO3; clone D19A7; Cell Signaling Technology), poly (ADP-ribose) polymerase 1 (PARP1; clone H-300; Santa Cruz Biotechnology), B-cell lymphoma 2 (clone C-2; Santa Cruz Biotechnology), HA-prove (clone Y-11; Santa Cruz Biotechnology), $\beta$-catenin (clone 14/Beta-Catenin; BD Biosciences, San Jose, CA), active $\beta$-catenin (clone 8E7; Millipore, Darmstadt, Germany), cyclin D1 (polyclonal; Cell Signaling Technology), NF-кB p50 (clone NLS; Santa Cruz Biotechnology), NF-кB p65 (polyclonal; Abcam, Cambridge, United Kingdom), matrix metallopeptidase 9 (MMP9; polyclonal; Thermo Fisher Scientific, Fremont, CA), and actin (clone AC-74; Sigma-Aldrich). Subcellular fractions of MCF7 cells were obtained using a Subcellular Protein Fractionation Kit (Thermo Scientific, Rockford, IL). Stepwise lysis of cells generated both functional cytoplasmic and nuclear protein extracts. The protocol was performed according to the manufacturer's instructions. Each fraction was separated by SDS-PAGE and a Western blot analysis was performed.

\section{Immunoprecipitation}

The Dynabeads-Protein A kit (Invitrogen) was used according to the manufacturer's protocol for immunoprecipitation (IP). In brief, polyclonal anti-CSNK2A1, antiSIRT6, or anti- $\beta$-catenin antibodies were cross-linked to Dynabeads-Protein A for 20 minutes at room temperature, and then the tube was placed on the magnet and the supernatant was removed. The tube was then incubated with rotation for 2 hours at $4^{\circ} \mathrm{C}$ for cell lysates to bind to the Dynabead-antibody complexes. CSNK2A1-, SIRT6-, or $\beta$-catenin-IP complexes were washed thrice with supplied washing buffer. Proteins were eluted by boiling in elution buffer and then processed for Western blot analysis.

\section{GST Pull-Down Assays}

To investigate a direct interaction between CSNK2A1 and SIRT6, we performed GST-mediated pull-down assays. The Pierce GST Protein Interaction Pull-Down kit (Thermo Scientific) was used according to the manufacturer's protocol to investigate a direct interaction between CSNK2A1 and SIRT6. The GST-fusion CSNK2A1 expression vector was kindly provided by Y.G. Yu (Department of Chemistry, Kookmin University, Korea). ${ }^{41}$ Recombinant GST-CSNK2A1 protein was expressed and purified from Cosmo Genetech Co. Ltd. SIRT6 proteins were expressed in 293 cells by transfection, followed by lysis in Pull-Down Lysis Buffer (Thermo Scientific). Briefly, $150 \mu \mathrm{g}$ of purified GST-CSNK2A1 fragment was bound to $50 \mu \mathrm{L}$ of glutathione agarose resin as a GST-fusion protein for 2 hours at $4^{\circ} \mathrm{C}$ and incubated with SIRT6-overexpressing cell lysates for 2 hours at $4^{\circ} \mathrm{C}$. After extensive washing with assay buffer containing $25 \mathrm{mmol} / \mathrm{L}$ Tris, $150 \mathrm{mmol} /$ $\mathrm{L} \mathrm{NaCl}$, and $2 \mathrm{mmol} / \mathrm{L}$ dithiothreitol $(\mathrm{pH}$ 8.0), the complex was eluted with $5 \mathrm{mmol} / \mathrm{L}$ reduced glutathione, and the pull-down products were separated by SDS-PAGE.

\section{In Vitro Kinase Assay}

To evaluate in vitro phosphorylation of SIRT6 by CSNK2A1, GST-CSNK2A1-tagged recombinant proteins $(4 \mu \mathrm{g})$ were incubated with purified His-SIRT6 (SIRT6-WT, SIRT6-T294A, SIRT6-S303A, SIRT6-S330A, or SIRT6$S 338 A$ mutant construct) recombinant proteins $(8 \mu \mathrm{g})$ in kinase buffer $(20 \mathrm{mmol} / \mathrm{L}$ Tris- $\mathrm{HCl}, 50 \mathrm{mmol} / \mathrm{L} \mathrm{KCl}, 10$ $\mathrm{mmol} / \mathrm{L} \mathrm{MgCl}_{2}$ [pH 8.0], $2.5 \mathrm{mmol} / \mathrm{L}$ cold ATP, and $1 \mu \mathrm{Ci}$ $\left.\left[\gamma-{ }^{32} \mathrm{P}\right] \mathrm{ATP}\right)$ for 30 minutes at $30^{\circ} \mathrm{C}$. Recombinant His-SIRT6 protein was expressed and purified from Cosmo Genetech Co. Ltd. The reaction products were separated on SDS-PAGE, and ${ }^{32} \mathrm{P}$-labeled proteins were detected by autoradiography using FUJIX BAS-2500 bioimaging analyzer (Fuji, Tokyo, Japan).

\section{Immunofluorescence Staining}

For the immunofluorescence staining, MCF7 cells were fixed with methanol and incubated with CSNK2A1 (dilution 1:50; clone 1AD9; Santa Cruz Biotechnology), SIRT6 (dilution 1:50; clone D8D12; Cell Signaling Technology), and pSIRT6 (Ser338) (dilution 1:100; polyclonal; Bioss Antibodies), $\beta$-catenin (dilution 1:100; BD Biosciences), and active $\beta$-catenin (dilution 1:100; Merck Millipore, Darmstadt, Germany) antibodies. Thereafter, the slides were incubated with Alexa Fluor 488 anti-mouse IgG or Alexa Fluor 594 anti-rabbit IgG (Invitrogen) and counterstained with DAPI. Images were taken with a Zeiss LSM 510 confocal microscope (Carl Zeiss, Göttingen, Germany). 


\section{Cell Cycle Analysis}

Transfected MCF7 or MDA-MB-231 cells, which contained dead cells, were harvested, washed with phosphate-buffered saline, and fixed in $70 \%$ ethanol at $4^{\circ} \mathrm{C}$ overnight. Thereafter, the cells were incubated with phosphate-buffered saline containing $50 \mu \mathrm{g} / \mathrm{mL}$ propidium iodide (Sigma-Aldrich) and $50 \mu \mathrm{g} / \mathrm{mL}$ RNase A (Sigma-Aldrich) at $37^{\circ} \mathrm{C}$ for 30 minutes. DNA content was measured by a FACStar flow cytometer (Becton-Dickinson, San Jose, CA) and analyzed using FlowJo software version 10.0 (Tree Star, Ashland, OR).

\section{In Vitro Migration and Invasion Assays}

A 24-transwell migration assay (Corning Life Sciences, Acton, MA) was performed to measure cell migration ability. Before seeding of cells, Transwell chambers were rehydrated for overnight, and MCF7 $\left(1 \times 10^{5}\right)$, T47D $\left(1 \times 10^{5}\right)$, or MDA-MB-231 $\left(5 \times 10^{4}\right)$ cells in the serum-free RPMI 1640 or DMEM were added to Transwell upper chambers. Media containing 20\% FBS was used in the lower chamber as a chemoattractant. After 24 hours, chambers were fixed and stained with Diff-Quick solution kit. The invasion assay was performed using a Transwell bioCoat Matrigel Invasion chamber (BD Biosciences). The MCF7 $\left(3 \times 10^{5}\right)$, T47D $\left(3 \times 10^{5}\right)$, or MDA-MB-231 cells $\left(1 \times 10^{5}\right)$ in RPMI 1640 or DMEM with $2 \%$ FBS were added to a Matrigel-coated chamber. Media containing 20\% FBS was used in the lower chamber as a chemoattractant. After incubation for 48 hours, chambers were fixed and stained with a Diff-Quick solution kit. The cells which had migrated or invaded to the lower surface of the filter were counted in five microscopic fields (magnification, $\times 100$ ) per well.

\section{Quantitative Reverse-Transcription Polymerase Chain Reaction}

RNA was isolated with the RNeasy Mini Kit (Qiagen Sciences, Valencia, CA), and reverse transcription of 1.5 $\mu \mathrm{g}$ of RNA was performed with TaqMan Reverse Transcription Reagents (Applied Biosystems, Foster City, CA). Quantitative reverse-transcription polymerase chain reaction was performed using the Applied Biosystems Prism 7900HT Sequence Detection System and Sybr Green polymerase chain reaction Master Mix (Applied Biosystems). All experiments were performed in triplicate, and the results were normalized to the expression of the glyceraldehyde-3-phosphate dehydrogenase reference housekeeping gene. Primer sequences for quantitative reverse-transcription polymerase chain reaction are listed in Table 1.

\section{Statistical Analysis}

The prognosis of BCA patients was evaluated by the survival analysis for the overall survival (OS) and the relapse-free survival (RFS). The follow-up end point was the date of the last contact or the date of death through

Table 1 The Primer Sequences Used for the Quantitative Real-Time Polymerase Chain Reaction

\begin{tabular}{|c|c|c|c|}
\hline Gene & Primer sequence forward/reverse & Product size & Accession number \\
\hline \multirow[t]{2}{*}{ CSNK2A1 } & F: 5'-GAACGCTTTGTCCACAGTGA-3' & 77 & NM_177559.2 \\
\hline & R: 5'-TATCGCAGCAGTTTGTCCAG-3' & & \\
\hline \multirow[t]{2}{*}{ SIRT6 } & F: 5'-AGGATGTCGGTGAATTACGC-3' & 261 & NM_016539.2 \\
\hline & R: 5'-AAAGGTGGTGTCGAACTTGG-3' & & \\
\hline \multirow[t]{2}{*}{ FOXO3 } & F: 5'-CGTAGTGAACTCATGGATGC-3' & 161 & NM_001455.3 \\
\hline & R: 5'-АСТTCСССТTССТСАGTGAT-3' & & \\
\hline \multirow[t]{2}{*}{ PARP1 } & F: 5'-GACCTGAAGGAGCTACTCATC-3' & 247 & NM_001618.3 \\
\hline & R: 5'-TTTCTCGGAATTCCTTTGGGG-3' & & \\
\hline \multirow[t]{2}{*}{ MMP9 } & F: 5'-GACGCAGACATCGTCATCCA-3' & 200 & NM_004994.2 \\
\hline & R: 5'-GCCGCGCCATCTGCGTTTCCAAA-3' & & \\
\hline CTNNB1 ( $\beta$-catenin) & F: 5'-AAAATGGCAGTGCGTTTAG-3' & 100 & NM_001904.3 \\
\hline NFKB1 (NF-кB p50) & R: 5'-ATGGGGCATTTTGTTGAGAG-3' & & \\
\hline \multirow[t]{2}{*}{ RELA (NF-кB p65) } & F: 5'-CTGAACCAGGGCATACCTGT-3' & 197 & NM_021975.3 \\
\hline & R: 5'-GAGAAGTCCATGTCCGCAAT-3' & & \\
\hline \multirow[t]{2}{*}{ GAPDH } & F: 5'-AACAGCGACACCCACTCCTC-3' & 258 & NM_001256799.1 \\
\hline & R: 5'-GGAGGGGAGATTCAGTGTGGT-3' & & \\
\hline
\end{tabular}

Web link to accession numbers: https://www.ncbi.nlm.nih.gov/gene.

$F$, forward; $R$, reverse. 
June 2013. OS was calculated from the date of diagnosis to the date of death by BCA or last contact. Patients who were alive at last contact or died of other causes were treated as censored for OS analysis. RFS was calculated from the date of diagnosis to the date of relapse of any type, death from BCA, or last contact. Patients who were alive at last contact and who had not experienced relapse of the tumor or died by other causes were treated as censored for RFS analysis. Survival analysis was evaluated by Kaplan-Meier survival analysis and Cox proportional hazards regression analyses. To evaluate the values between the groups, Pearson's $\chi^{2}$ test and the Student's $t$-test were used. All experiments were performed three times, and representative data are presented. Statistical analysis was performed using SPSS software version 19.0 (IBM, San Jose, CA). The $P$ values $<0.05$ were considered statistically significant.

\section{Results}

Expression of CSNK2A1, SIRT6, and pSIRT6 Predicts Shorter Survival of BCA Patients

First, we investigated the expression of CSNK2A1, SIRT6, and pSIRT6 by immunohistochemical staining of BCA samples. CSNK2A1, SIRT6, and pSIRT6 were expressed in both the cytoplasm and nuclei of tumor cells (Figure 1A). The cutoff for the immunohistochemical staining score for CSNK2A1 was 7, and the cutoff was 6 for both nuclear SIRT6 and cytoplasmic SIRT6 expression. The cutoffs for nuclear pSIRT6 and cytoplasmic pSIRT6 were 9 and 5, respectively. The expression of CSNK2A1 was considered positive when a combined score was $\geq 7$. Nuclear SIRT6 and cytoplasmic SIRT6 expression were considered positive when a combined score was $\geq 6$. Nuclear pSIRT6 and cytoplasmic pSIRT6 expression were considered positive when a combined score
A

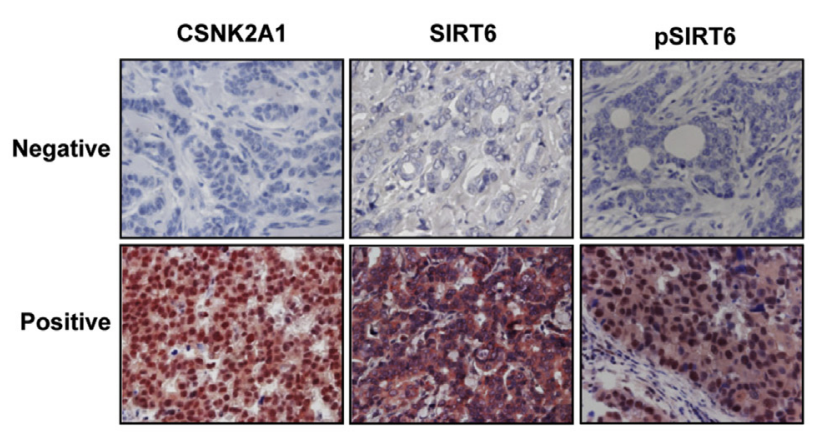

C
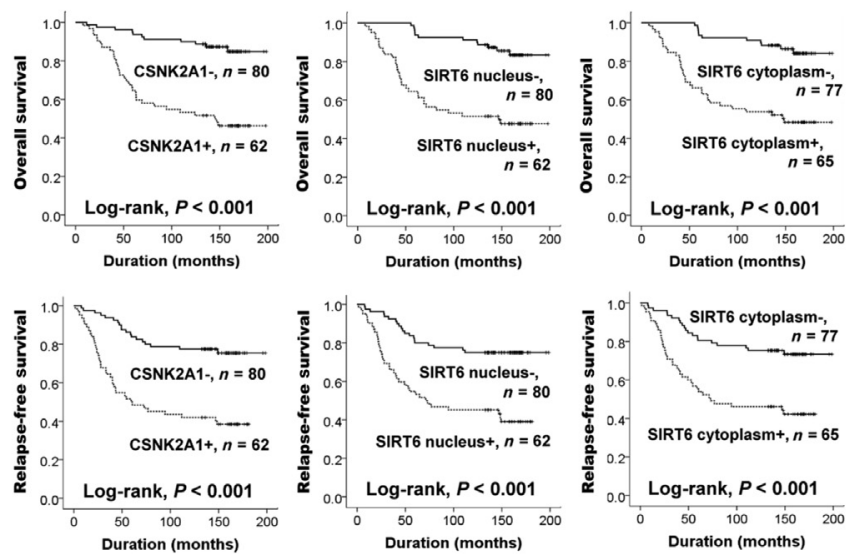

B

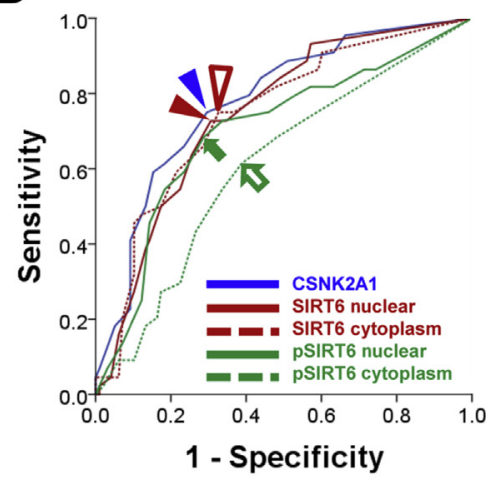

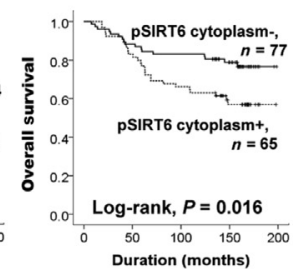
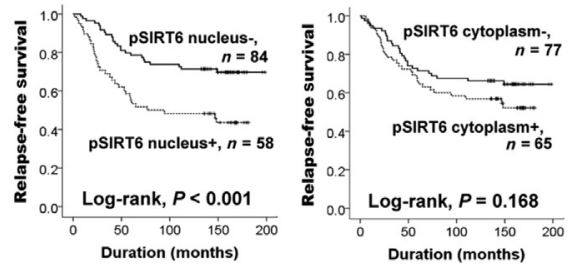

Figure 1 Expression of CSNK2A1, SIRT6, and pSIRT6 in human breast carcinomas and Kaplan-Meier survival analysis in 142 breast carcinoma patients. A: Immunohistochemical expression of CSNK2A1, SIRT6, and pSIRT6 in breast carcinoma. CSNK2A1, SIRT6, and pSIRT6 are expressed in both the cytoplasm and nuclei of tumor cells. B: Statistical analysis of the sensitivity and specificity of the immunohistochemical staining scores of nuclear CSNK2A1, nuclear SIRT6, cytoplasmic SIRT6, nuclear pSIRT6, and cytoplasmic pSIRT6 for death in breast carcinoma patients by receiver operator characteristic curves. The blue arrowhead indicates the cutoff for the immunohistochemical staining score of CSNK2A1, and the red filled arrowhead indicates the cutoff for nuclear expression of SIRT6 immunostaining. The red empty arrowhead indicates the cutoff for the cytoplasmic expression of SIRT6 immunostaining. The green filled arrow and green empty arrow indicate the cutoffs for nuclear and cytoplasmic expression of pSIRT6, respectively. C: Overall survival and relapse-free survival according to CSNK2A1 expression, nuclear SIRT6 expression, cytoplasmic SIRT6 expression, nuclear pSIRT6 expression, and cytoplasmic pSIRT6 expression. Original magnification, $\times 400($ A). CSNK2A1, protein kinase CK2 $\alpha 1$; $p$, phosphorylated; SIRT6, sirtuin 6 . 
was $\geq 9$ and 5, respectively (Table 2, Figure 1B). In BCAs, $44 \%, 44 \%, 41 \%$, and $46 \%$ of BCA were classified as CSNK2A1-positive, nuclear SIRT6-positive, cytoplasmic SIRT6-positive, nuclear pSIRT6-positive, and cytoplasmic pSIRT6-positive, respectively. CSNK2A1-positivity was significantly associated with distant metastatic relapse $(P<0.001)$ and HER2 expression $(P=0.004)$ and negatively correlated with PR expression $(P=0.005)$. In addition, CSNK2A1 expression was significantly associated with nuclear and cytoplasmic expression of SIRT6 $(P<0.001)$ and nuclear and cytoplasmic expression of pSIRT6 $(P<0.001)$. Seventy-one percent of BCAs expressing nuclear SIRT6 or nuclear pSIRT6 also expressed CSNK2A1. The expression of both nuclear and cytoplasmic SIRT6 was significantly associated with distant metastatic relapse, increased mitotic count, higher histologic grade, and HER2 expression. SIRT6 expression was negatively correlated with ER and PR expression. The nuclear expression of pSIRT6 was significantly associated with age of patients, distant metastatic relapse, higher histologic grade, HER2 expression, and loss of ER expression. Cytoplasmic expression of pSIRT6 was significantly associated with increased mitotic count, higher histologic grade, HER 2 expression, and loss of ER expression (Table 3). In addition, when we investigated the public data for the expression of CSNK2A1 and SIRT6 in breast tissue using the Oncomine database, ${ }^{42} 5$ of 65 data sets for CSNK2A1 expression and 5 of 56 data sets for SIRT6 expression matched our search parameters (gene name: CSNK2A1 or SIRT6; tissue: breast; comparison: normal versus carcinoma; significance: $P<0.001)$. The expression of CSNK2A1 mRNA was significantly increased in BCAs compared with normal breast tissue in four data sets and decreased in one data set (Supplemental Figure S1A). The expression of SIRT6 mRNA was significantly increased in invasive BCAs compared with normal breast tissue in five data sets (Supplemental Figure S1A).

Table 2 Statistical Analysis to Determine the Cutoffs for the Immunohistochemical Staining for CSNK2A1, SIRT6, and pSIRT6

\begin{tabular}{llll}
\hline Expression & Cutoff & AUC & $P$ \\
\hline CSNK2A1 & All & 0.779 & $<0.001$ \\
& $\geq 7$ & 0.727 & $<0.001$ \\
SIRT6 nuclear & All & 0.746 & $<0.001$ \\
\multirow{2}{*}{ SIRT6 cytoplasm } & $\geq 6$ & 0.711 & $<0.001$ \\
& All & 0.744 & $<0.001$ \\
pSIRT6 nuclear & $\geq 6$ & 0.712 & $<0.001$ \\
& All & 0.702 & $<0.001$ \\
pSIRT6 cytoplasm & $\geq 9$ & 0.698 & $<0.001$ \\
& All & 0.61 & 0.036 \\
& $\geq 5$ & 0.613 & 0.032 \\
\hline
\end{tabular}

Statistical analysis of the sensitivity and specificity of the immunohistochemical staining scores of the nuclear CSNK2A1, nuclear SIRT6, cytoplasmic SIRT6, nuclear pSIRT6, and cytoplasmic pSIRT6 expression for the death of the breast carcinoma patients by receiver operator characteristic curves.

AUC, area under curve; pSIRT6, phosphorylated sirtuins 6 .
Univariate Cox regression analysis revealed age $(P<0.001)$; tumor stage $(P=0.023)$; histologic grade $(P=0.017)$; and the expression of HER2 $(P=0.003)$, CSNK2A1 $(P<0.001)$, nuclear SIRT6 $(P<0.001)$, cytoplasmic SIRT6 $(P<0.001)$, nuclear pSIRT6 $(P<0.001)$, and cytoplasmic pSIRT6 $(P=0.018)$ were significantly associated with OS of BCA patients. The factors significantly associated with RFS in the univariate analysis were age $(P=0.024)$ and the expression of HER2 $(P=0.011)$, CSNK2A1 $(P<0.001)$, nuclear SIRT6 $(P<0.001)$, cytoplasmic SIRT6 $(P<0.001)$, and nuclear pSIRT6 $(P=0.001)$ (Table 4, Figure 1C). Multivariate analysis performed included age of the patients; tumor stage; histologic grade; and the expression of HER2, ER, PR, CSNK2A1, nuclear SIRT6, cytoplasmic SIRT6, nuclear pSIRT6, and cytoplasmic pSIRT6. On multivariate survival analysis, age of the patients $(P=0.034)$, tumor stage $(P=0.035)$, and the expression of CSNK2A1 $(P=0.008)$ and nuclear SIRT6 $(P=0.005)$ were independent prognostic indicators for OS of BCA patients. The factors significantly associated with RFS by multivariate analysis were the expression of CSNK2A1 $(P=0.002)$ and nuclear SIRT6 $(P=0.016)$. The expression of CSNK2A1 predicted a 2.821 -fold [ $95 \%$ confidence interval (CI), 1.310- to 6.074-fold] greater risk of death and a 2.595 -fold $(95 \% \mathrm{CI}$, 1.406- to 4.788 -fold) greater risk of relapse or death. Nuclear expression of SIRT6 also indicated a 2.985 -fold $(95 \%$ CI, 1.398- to 6.373-fold) greater risk of death and a 2.105 -fold (95\% CI, 1.150- to 3.853-fold) greater risk of relapse or death (Table 4). In addition, because the expression of SIRT6 and pSIRT6 were closely correlated, we performed additional multivariate analysis excluding the expression of nuclear and cytoplasmic expression of SIRT6 (Table 4). Multivariate analysis revealed nuclear expression of pSIRT6 as an independent indicator of poor prognosis for OS for BCA patients $(P=0.019$; hazard ratio, $2.247 ; 95 \% \mathrm{CI}, 1.140-4.431)$.

Inhibition of CSNK2A1 Decreases the Proliferation and the Invasiveness of Breast Cancer Cells

Because the expression of CSNK2A1 predicted poor survival of BCA patients, we investigated the effect of the inhibition of CSNK2A1 for the proliferation of two BCA cell lines (MCF7 and T47D). The treatment of CSNK2 inhibitors, CX4945 and emodin, and knockdown of CSNK2Al with siRNA for CSNK2A1 inhibited the proliferation of BCA cells (Figure 2, $A-C)$. In flow cytometric cell cycle analysis, the sub- $G_{1}$ and $\mathrm{G}_{0} / \mathrm{G}_{1}$ population was increased with knockdown of CSNK2A1 in MCF7 cells (Figure 2D). In addition, the knockdown of CSNK2Al significantly inhibited migration and invasion of two BCA cell lines (Figure 2, E and F).

\section{CSNK2A1 Binds to SIRT6 and Phosphorylates Ser338 on SIRT6 in Breast Cancer Cells}

Because the expression of CSNK2A1 promoted the proliferation of cells and correlated with the expression of SIRT6 
Table 3 Association of the Expression of CSNK2A1, SIRT6, and pSIRT6 with Clinicopathologic Factors

\begin{tabular}{|c|c|c|c|c|c|c|c|c|c|c|c|}
\hline \multirow[b]{2}{*}{ Characteristics } & \multirow[b]{2}{*}{ No. } & \multicolumn{2}{|l|}{ CSNK2A1 } & \multicolumn{2}{|c|}{ SIRT6 nuclear } & \multicolumn{2}{|c|}{ SIRT6 cytoplasm } & \multicolumn{2}{|c|}{ pSIRT6 nuclear } & \multicolumn{2}{|c|}{ pSIRT6 cytoplasm } \\
\hline & & Positive & $P$ & Positive & $P$ & Positive & $P$ & Positive & $P$ & Positive & $P$ \\
\hline \multicolumn{12}{|l|}{ Age, years } \\
\hline$<50$ & 101 & $39(39)$ & 0.057 & $39(39)$ & 0.057 & $41(41)$ & 0.052 & $35(35)$ & 0.018 & $43(43)$ & 0.230 \\
\hline$\geq 50$ & 41 & $23(56)$ & & $23(56)$ & & $24(59)$ & & $23(56)$ & & $22(54)$ & \\
\hline \multicolumn{12}{|l|}{ TNM stage } \\
\hline I & 26 & $11(42)$ & 0.403 & $15(58)$ & 0.069 & $16(62)$ & 0.009 & $11(42)$ & 0.089 & $15(58)$ & 0.148 \\
\hline II & 97 & $40(41)$ & & $36(37)$ & & $36(37)$ & & $35(36)$ & & $39(40)$ & \\
\hline III and IV & 19 & $11(58)$ & & $11(58)$ & & $13(68)$ & & $12(63)$ & & $11(58)$ & \\
\hline \multicolumn{12}{|l|}{ T stage } \\
\hline 1 & 38 & $17(45)$ & 0.985 & $21(55)$ & 0.144 & $21(55)$ & 0.275 & $14(37)$ & 0.589 & $17(45)$ & 0.830 \\
\hline 2 & 95 & $41(43)$ & & $36(38)$ & & $39(41)$ & & $38(40)$ & & $43(45)$ & \\
\hline 3 and 4 & 9 & $4(44)$ & & $5(56)$ & & $5(56)$ & & $5(56)$ & & $5(56)$ & \\
\hline \multicolumn{12}{|l|}{ LN metastasis } \\
\hline Absence & 80 & $31(39)$ & 0.180 & $33(41)$ & 0.510 & $33(41)$ & 0.219 & $28(35)$ & 0.107 & $35(44)$ & 0.582 \\
\hline Presence & 62 & $31(50)$ & & $29(47)$ & & $32(52)$ & & $30(48)$ & & $30(48)$ & \\
\hline \multicolumn{12}{|c|}{ Distant metastatic relapse } \\
\hline Absence & 107 & $35(33)$ & $<0.001$ & $39(36)$ & 0.002 & $41(38)$ & 0.002 & $35(33)$ & $<0.001$ & $44(41)$ & 0.052 \\
\hline Presence & 35 & $27(77)$ & & $23(66)$ & & $24(69)$ & & $23(66)$ & & $21(60)$ & \\
\hline \multicolumn{12}{|l|}{ Histologic type } \\
\hline NST & 134 & $57(43)$ & 0.269 & $59(44)$ & 0.718 & $62(46)$ & 0.629 & $54(40)$ & 0.588 & $62(46)$ & 0.629 \\
\hline Lobular & 8 & $5(63)$ & & $3(38)$ & & $3(38)$ & & $4(50)$ & & $3(38)$ & \\
\hline \multicolumn{12}{|l|}{ Mitoses/10 HPF } \\
\hline $0-9$ & 91 & $35(38)$ & 0.073 & $33(36)$ & 0.017 & $33(36)$ & 0.004 & $32(35)$ & 0.143 & $34(37)$ & 0.024 \\
\hline $10-19$ & 26 & $11(42)$ & & $12(46)$ & & $14(54)$ & & $12(46)$ & & $15(58)$ & \\
\hline$>19$ & 25 & $16(64)$ & & $17(68)$ & & $18(72)$ & & $14(56)$ & & $16(64)$ & \\
\hline \multicolumn{12}{|l|}{ Histologic grade } \\
\hline 1 & 49 & $16(33)$ & 0.120 & $16(33)$ & 0.017 & $16(33)$ & 0.003 & $12(24)$ & 0.015 & $13(27)$ & 0.002 \\
\hline 2 & 64 & $30(47)$ & & $27(42)$ & & $28(44)$ & & $31(48)$ & & $33(52)$ & \\
\hline 3 & 29 & $16(55)$ & & $19(66)$ & & $21(72)$ & & $15(52)$ & & $19(66)$ & \\
\hline \multicolumn{12}{|l|}{ HER2 } \\
\hline Negative & 98 & $35(36)$ & 0.004 & $34(35)$ & 0.001 & $37(38)$ & 0.004 & $29(30)$ & $<0.001$ & $36(37)$ & 0.001 \\
\hline Positive & 44 & $27(61)$ & & $28(64)$ & & $28(64)$ & & $29(66)$ & & $29(66)$ & \\
\hline \multicolumn{12}{|l|}{ ER } \\
\hline Negative & 63 & $33(52)$ & 0.061 & $33(52)$ & 0.061 & $36(57)$ & 0.015 & $32(51)$ & 0.031 & $36(57)$ & 0.015 \\
\hline Positive & 79 & $29(37)$ & & $29(37)$ & & $29(37)$ & & $26(33)$ & & $29(37)$ & \\
\hline \multicolumn{12}{|l|}{ PR } \\
\hline Negative & 59 & $34(58)$ & 0.005 & $34(58)$ & 0.005 & $34(58)$ & 0.017 & $25(42)$ & 0.755 & $31(53)$ & 0.172 \\
\hline Positive & 83 & $28(34)$ & & $28(34)$ & & $31(37)$ & & $33(40)$ & & $34(41)$ & \\
\hline pSIRT6 cytoplas & & & & & & & & & & & \\
\hline Negative & 77 & $21(27)$ & $<0.001$ & $24(31)$ & 0.001 & $22(29)$ & $<0.001$ & $9(12)$ & $<0.001$ & & \\
\hline Positive & 65 & $41(63)$ & & $38(58)$ & & $43(66)$ & & $49(75)$ & & & \\
\hline pSIRT6 nuclear & & & & & & & & & & & \\
\hline Negative & 84 & $21(25)$ & $<0.001$ & $23(27)$ & $<0.001$ & $24(29)$ & $<0.001$ & & & & \\
\hline Positive & 58 & $41(71)$ & & $39(67)$ & & $41(71)$ & & & & & \\
\hline SIRT6 cytoplasr & & & & & & & & & & & \\
\hline Negative & 77 & $16(21)$ & $<0.001$ & $3(4)$ & $<0.001$ & & & & & & \\
\hline Positive & 65 & $46(71)$ & & $59(91)$ & & & & & & & \\
\hline SIRT6 nuclear & & & & & & & & & & & \\
\hline Negative & 80 & $18(23)$ & $<0.001$ & & & & & & & & \\
\hline Positive & 62 & $44(71)$ & & & & & & & & & \\
\hline
\end{tabular}

Data for positive values are expressed as $n(\%)$.

CSNK2A1, protein kinase CK2 $\propto 1$; ER, estrogen receptor; HER2, human epidermal growth factor receptor 2; HPF, high-power field; LN, lymph node; NST, invasive carcinoma of no special type; p, phosphorylated; PR, progesterone receptor; SIRT6, sirtuin 6; TNM, staging notation system.

and pSIRT6 in human BCA samples, we investigated the effect of a knockdown of CSNK2A1 on signaling related with apoptosis of cells and the relationship between the expressions of CSNK2A1 and SIRT6 in MCF7 BCA cells.
CSNK2A1 affected the expression of apoptosis-related molecules; CSNK2Al knockdown increased expression of FOXO3 and PARP1 and decreased expression of B-cell lymphoma 2 (Figure 3, A and B). Interestingly, the 
Table 4 Clinicopathologic Factors and Their Effect on Overall Survival and Relapse-Free Survival by Univariate and Multivariate Cox Proportional Hazards Regression Analysis

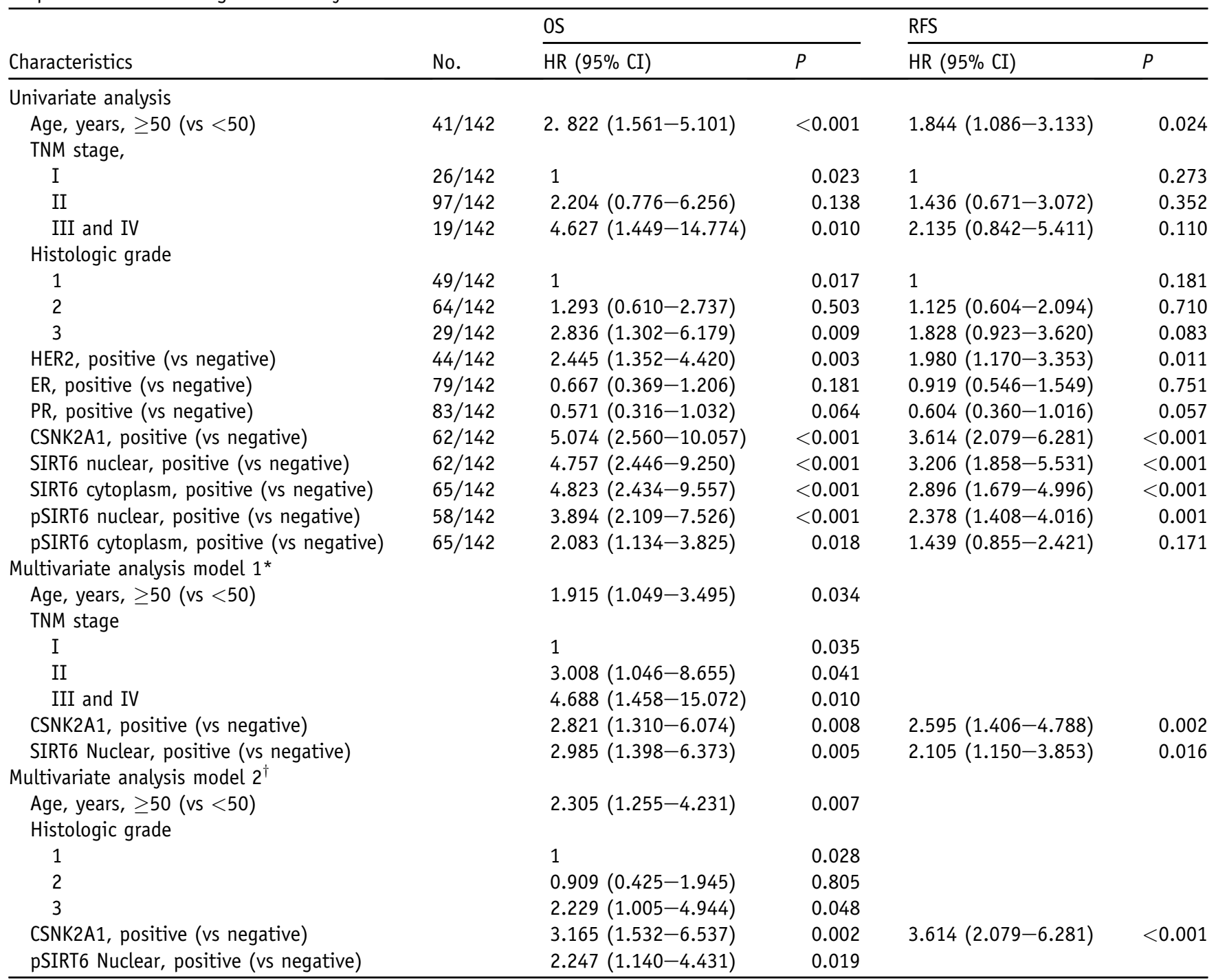

*Variables included in the multivariate analysis were age; TNM stage; histologic grade; and the expression of HER2, ER, PR, CSNK2A1, nuclear SIRT6, cytoplasmic SIRT6, nuclear pSIRT6, and cytoplasmic pSIRT6 (model 1).

†Variables included in the multivariate analysis were age; TNM stage; histologic grade; and the expression of HER2, ER, PR, CSNK2A1, nuclear pSIRT6, and cytoplasmic pSIRT6 (model 2).

CSNK2A1, protein kinase CK2 $\alpha 1$; ER, estrogen receptor; HER2, human epidermal growth factor receptor 2; HR, hazard ratio; 0S, overall survival; PR, progesterone receptor; $p$, phosphorylated; RFS, relapse-free survival; SIRT6, sirtuin 6; TNM, staging notation system.

phosphorylation of Ser338 on SIRT6 decreased with the knockdown of CSNK2A1 (Figure 3A). However, the expression of SIRT6 mRNA and protein were not changed significantly by a knockdown of CSNK2A1 (Figure 3B). Therefore, we investigated a direct relationship between CSNK2A1 and SIRT6. A co-IP experiment demonstrated that CSNK2A1 binds with SIRT6 (Figure 3C). SIRT6 and CSNK2A1 were detected by Western blot analysis after IP with either CSNK2A1 or SIRT6, respectively (Figure 3C). In addition, confocal microscopy images also showed that CSNK2A1 is colocalized with SIRT6 or pSIRT6 in both the nuclei and cytoplasm (Figure 3D). These findings suggest that CSNK2A1 binds to SIRT6 and might be involved in the regulation of phosphorylation of SIRT6. In addition, the phosphorylation of SIRT6-Ser338 decreased with the transfection of dominant negative $C S N K 2 A 1$ and increased with the transfection of WT CSNK2Al (Figure 3E). However, despite successful transfection of WT and mutant CSNK2Al as demonstrated by HA expression, the protein level of CSNK2A1 was unchanged. This phenomenon was reported previously with the use of HA-tagged WT CSNK2Al (pRc/ CMV_CSNK2A1-HA). ${ }^{4,43}$ However, the reason is not clear. Therefore, further study is needed. Further evidence of a CSNK2A1-SIRT6 interaction was found via a GST pulldown assay and an in vitro kinase assay. Direct binding of CSNK2A1 to SIRT6 was reproduced in vitro by a GST 

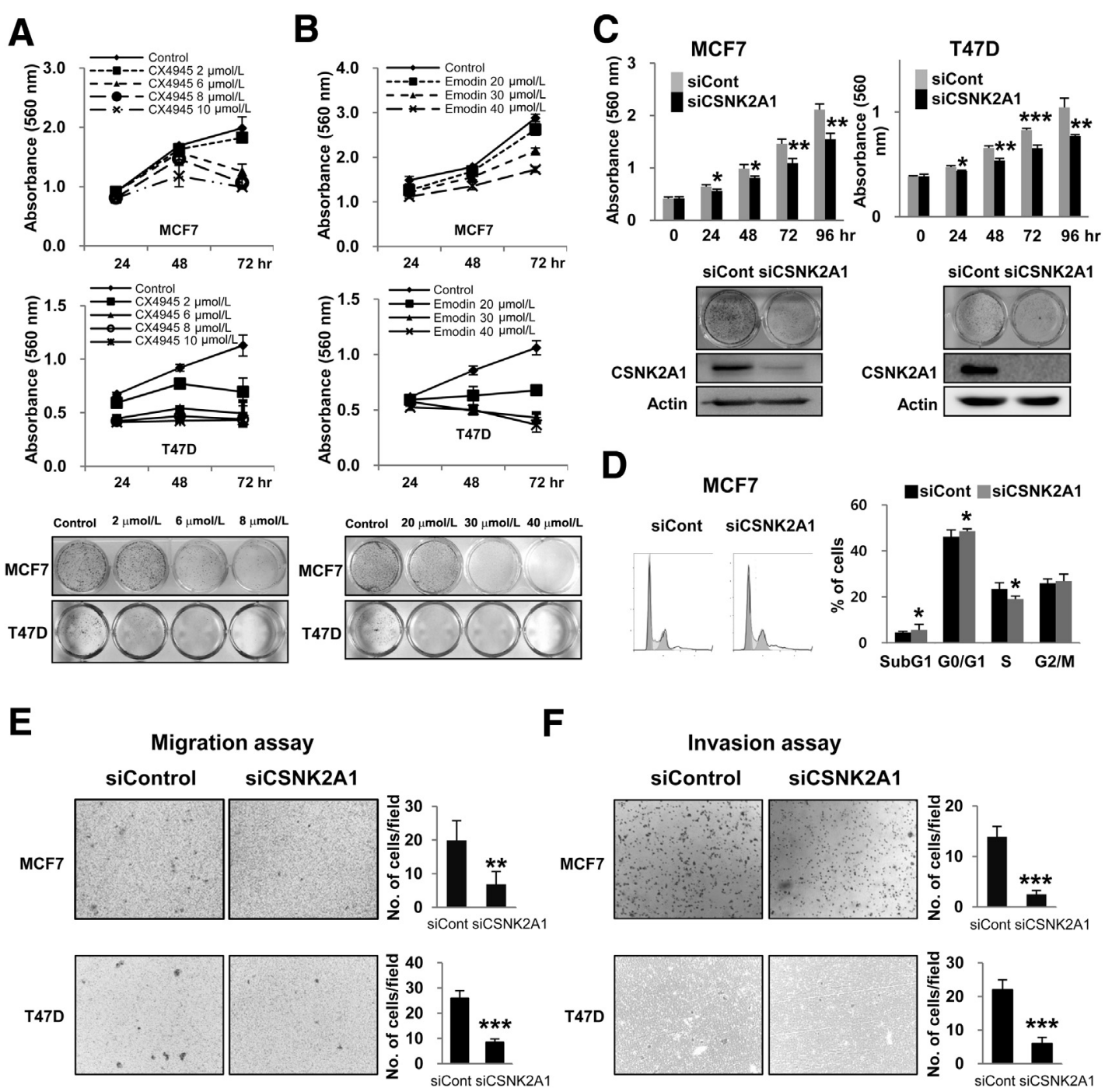

Figure 2 Inhibition of CSNK2A1 decreased the proliferation and invasiveness of breast cancer cells. A and B: Treatment with the CSNK2 inhibitors, CX4945 (A) and emodin (B), significantly inhibited the growth of both MCF7 and T47D cells in a dose- and time-dependent manner. C: The knockdown of CSNK2A1 inhibited the proliferation of both MCF7 and T47D cells as indicated by MTT and colony-forming assays. D: The sub- $\mathrm{G}_{1}$ and $\mathrm{G}_{0} / \mathrm{G}_{1}$ population increased with the knockdown of CSNK2A1 in flow cytometric cell cycle analysis. $\mathbf{E}$ and $\mathbf{F}$ : The knockdown of CSNK2A1 significantly reduced cell migration (E) and invasion (F) in both MCF7 and T47D cell lines. ${ }^{*} P<0.05,{ }^{* *} P<0.01$, and ${ }^{* * *} P<0.001$. CSNK2A1, protein kinase CK2 $\alpha 1$.

pull-down assay (Figure 3F). An in vitro kinase assay showed that CSNK2A1 phosphorylates WT SIRT6, SIRT6T294A mutant, SIRT6-S303A mutant, and SIRT6-S330A mutant proteins. However, the SIRT6-S338A mutant protein was not phosphorylated by CSNK2A1 (Figure 3G). These results show that CSNK2A1 is involved in the phosphorylation of SIRT6 on Ser338.

\section{Knockdown of SIRT6 Inhibited Proliferation and} Invasion of Breast Cancer Cells and Mutation of SIRT6 at the Ser338 Phosphorylation Site Attenuated the Proliferation of Breast Cancer Cells

Because the expression of both CSNK2A1 and SIRT6 correlated with poor prognosis of BCA patients and CSNK2A1 affected the phosphorylation of Ser338 on
SIRT6, we investigated the effects of a knockdown of SIRT6 and a mutation of SIRT6 at the Ser338 phosphorylation site. The knockdown of SIRT6 inhibited proliferation of MCF7, T47D, and MDA-MB 231 cell lines (Figure 4A), which correlated with $G_{0} / G_{1}$ and $G_{2} / M$ cell cycle arrest as indicated by flow cytometric analysis (Figure 4B). The knockdown of SIRT6 also inhibited migration and invasion of MCF7, T47D, and MDA-MB 231 cell lines (Figure 4, C and D). The decreased invasive potential of tumor cells with a knockdown of SIRT6 was associated with a decrease in the expression of MMP9 mRNA and protein (Figure 5, A and B). In addition, the expression of mRNAs and proteins involved in cellular proliferation, such as $\beta$-catenin, cyclin $\mathrm{D} 1$, and NF- $\kappa \mathrm{B}$, were decreased with a knockdown of SIRT6 (Figure 5, A and B). The protein levels of p53 and acetylated p53 increased with a knockdown of SIRT6 (Figure 5A). 
A

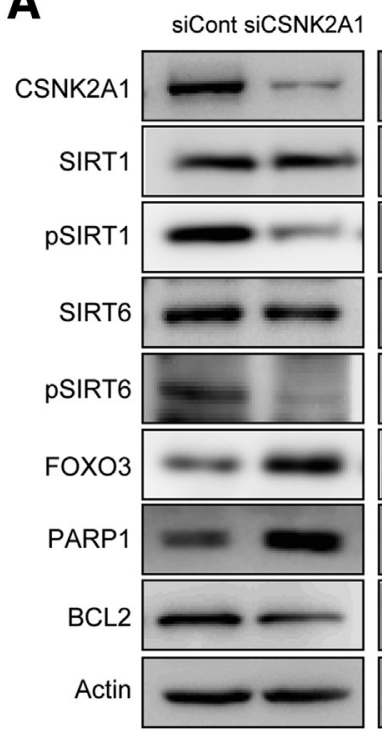

D
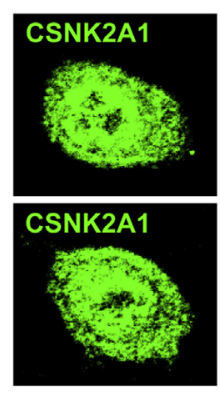

F

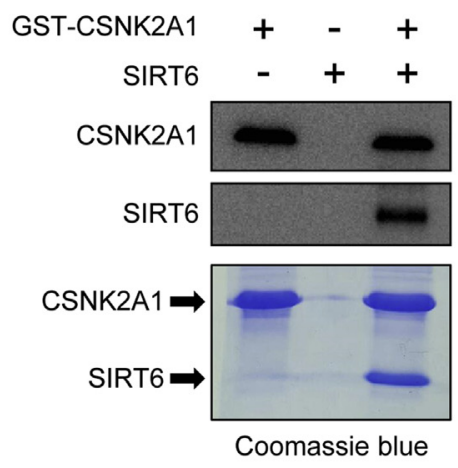

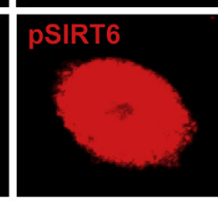

$+$
B
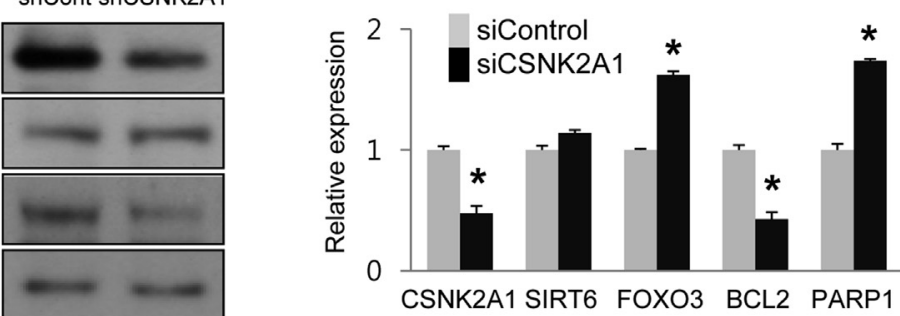

C

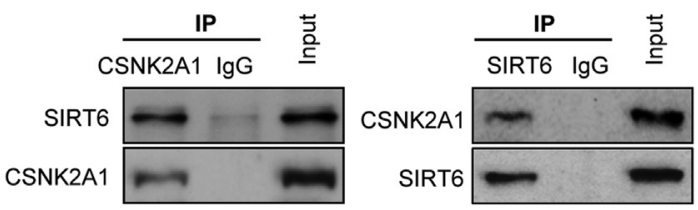

E
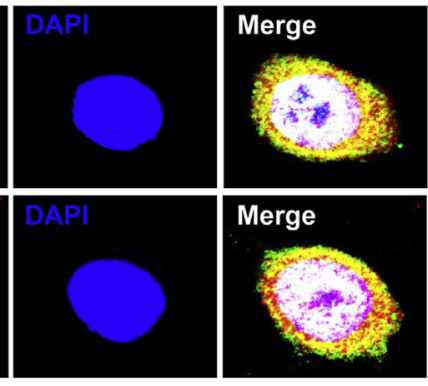

G

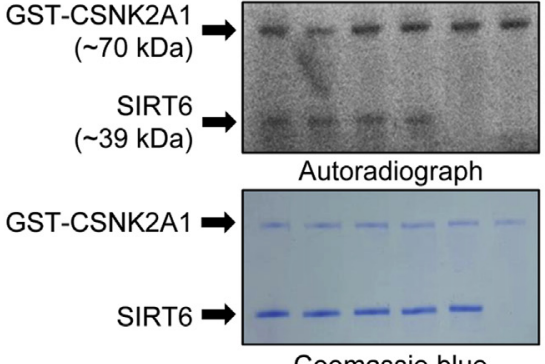

Coomassie blue

Figure 3 CSNK2A1 regulates the phosphorylation of SIRT6 in MCF7 cells. A and B: The protein expression of SIRT1 and SIRT6, and SIRT6 mRNA levels were not significantly changed with knockdown of CSNK2A1 in MCF7 cells. However, the protein levels of pSIRT1 and pSIRT6 decreased with knockdown of CSNK2A1 by siRNA or shRNA for CSNK2A1 in MCF7 cells. In addition, the knockdown of CSNK2A1 increased protein and mRNA levels of F0X03 and PARP1 but decreased protein and mRNA levels of BCL2. C: SIRT6 is detected in samples immunoprecipitated for CSNK2A1 and vice versa. D: Confocal microscopic images after immunofluorescence staining of CSNK2A1 and SIRT6 or pSIRT6 shows colocalization of CSNK2A1 and SIRT6 or pSIRT6 both in the cytoplasm and nuclei (colocalization is yellow in the cytoplasm and white in the nuclei in the merged images). E: Western blot analysis of CSNK2A1, SIRT6, pSIRT6, SIRT1, and pSIRT1. MCF7 cells were transfected with control vector (pFLAG-CMV-2), HA-tagged mutant CSNK2A1 vector (pRc/CMV_CSNK2A1_K68M-HA), or HA-tagged wild-type CSNK2A1 vector (pRc/CMV_CSNK2A1-HA). pSIRT6 was decreased with transfection of mutant CSNK2A1 and increased with transfection of wild-type CSNK2A1. F: A GST pulldown assay indicates direct binding of CSNK2A1 to SIRT6. G: An in vitro kinase assay indicates that CSNK2A1 phosphorylates wild-type SIRT6, SIRT6-T294A, SIRT6-S303A, and SIRT6-S330A mutant proteins. However, the SIRT6-S338A mutant protein was not phosphorylated by CSNK2A1. ${ }^{*} P<0.05$. BCL2, B-cell lymphoma 2; CSNK2A1, protein kinase CK2 $\alpha 1$; F0X03, forkhead box 03; GST, glutathione S-transferase; p, phosphorylated; PARP1, poly (ADP-ribose) polymerase 1; SIRT6, sirtuin 6. 
A
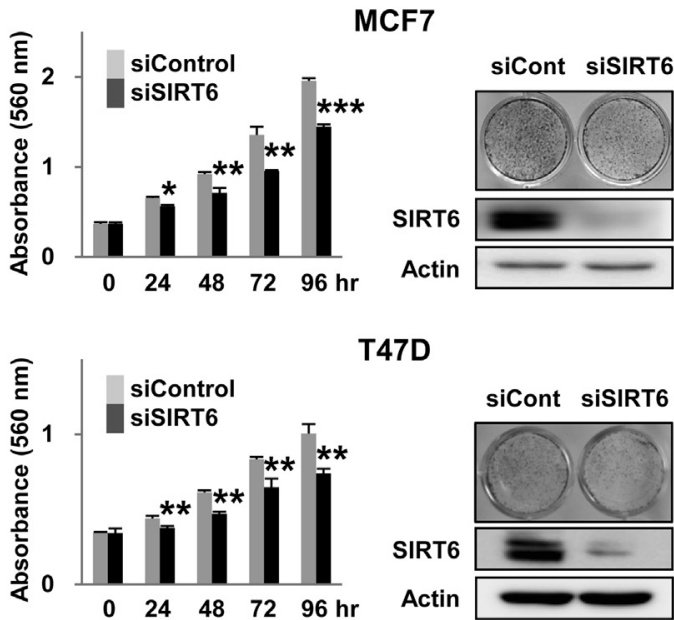

T47D
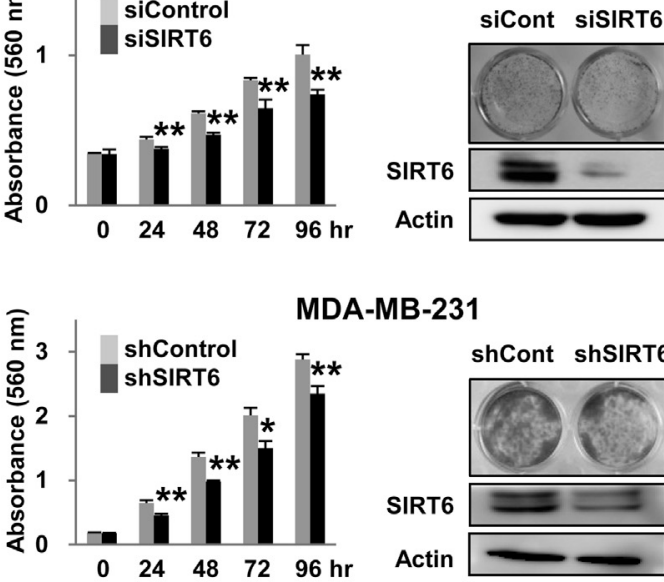

MDA-MB-231

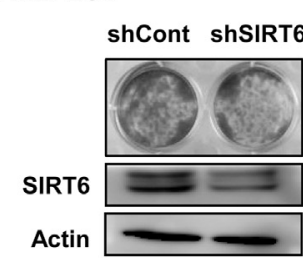

B

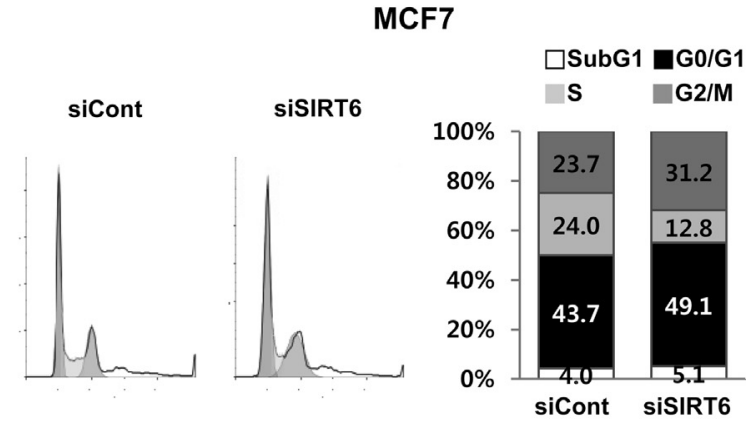

MDA-MB-231

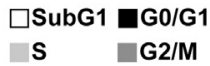

shCont

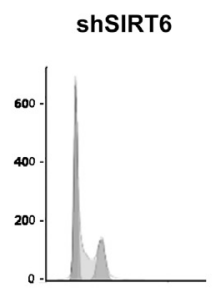

C

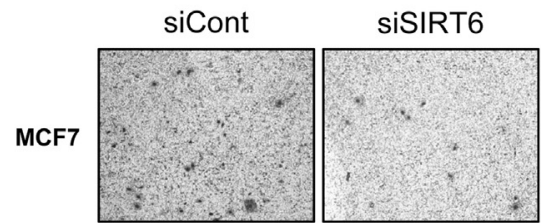

siCont

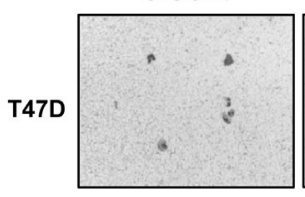

shCont

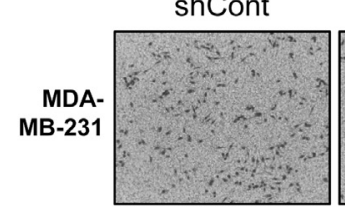

siSIRT6

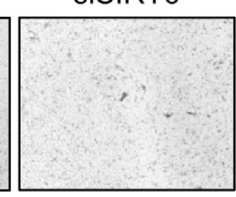

shSIRT6

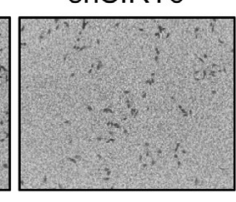

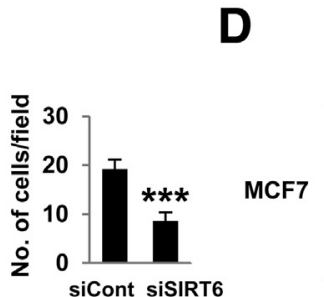$$
\text { 的 }
$$

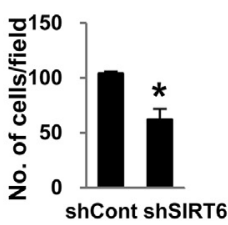

T47D

Invasion assay
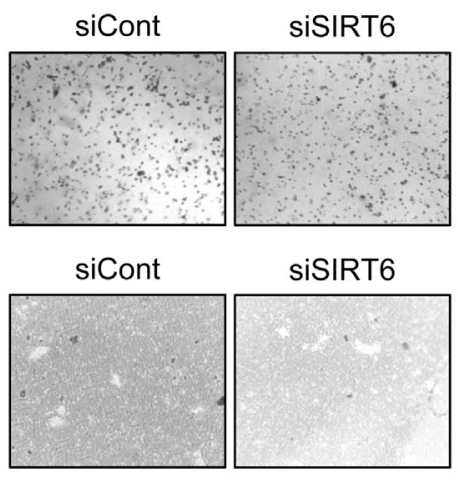

shCont

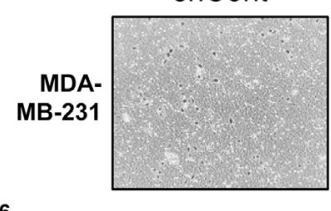

siSIRT6
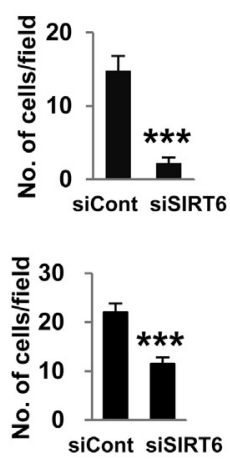

shSIRT6
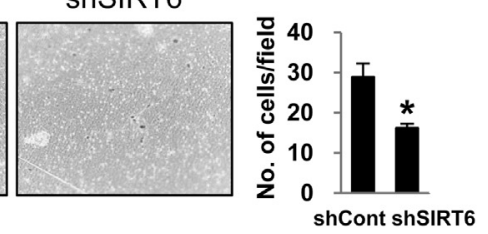

Figure 4 The expression of SIRT6 is associated with the proliferation and invasiveness of breast cancer cells. A: The knockdown of SIRT6 significantly inhibited the growth of MCF7, T47D, and MDA-MB 231 cell lines as indicated with MTT and colony-forming assays. B: The knockdown of SIRT6 induced $\mathrm{G}_{0} / \mathrm{G}_{1}$ arrest in both MCF7 and MDA-MB 231 cells as indicated by flow cytometric cell cycle analysis. C and D: The knockdown of SIRT6 significantly inhibited migration (C) and invasion activity (D) of MCF7, T47D, and MDA-MB 231 cell lines. ${ }^{*} P<0.05,{ }^{* *} P<0.01$, and ${ }^{* * *} P<0.001$. SIRT6, sirtuin 6.

Thereafter, we investigate the effects of the phosphorylation of SIRT6 in MCF7 cells. The proliferation of MCF7 cells was increased when transfected with WT SIRT6; however, the proliferation of MCF7 cells decreased with transfection of SIRT6-S338A mutant (Figure 5C). The transfection of WT SIRT6 increased expression of protein of SIRT6, pSIRT6, MMP9, active $\beta$-catenin, cyclin D1, and NF- $\kappa$ B (Figure 5D). In contrast, pSIRT6 was decreased with transfection of SIRT6-S338A mutant despite the significant increase of protein expression of SIRT6 (Figure 5D). 
A

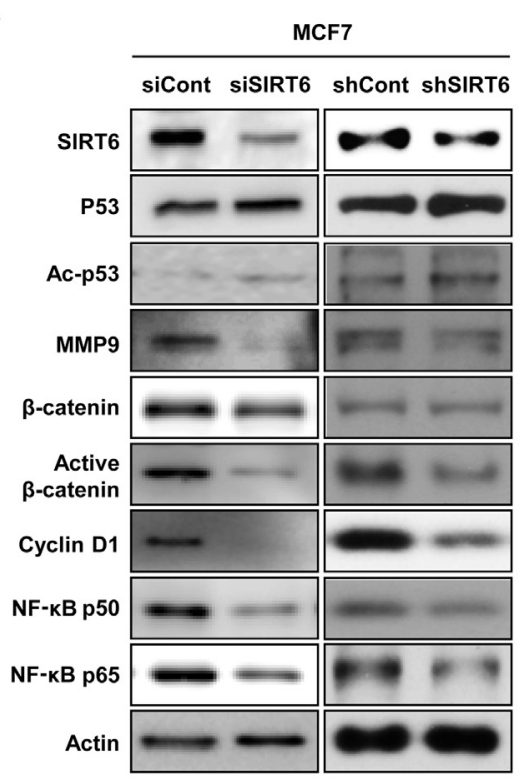

C

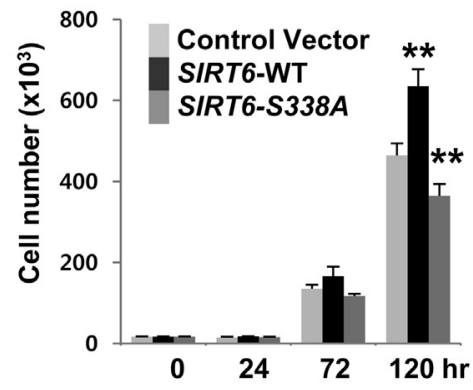

MDA-MB-231

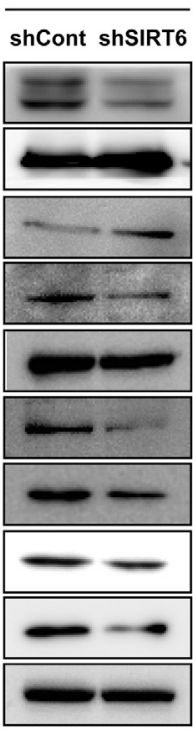

B
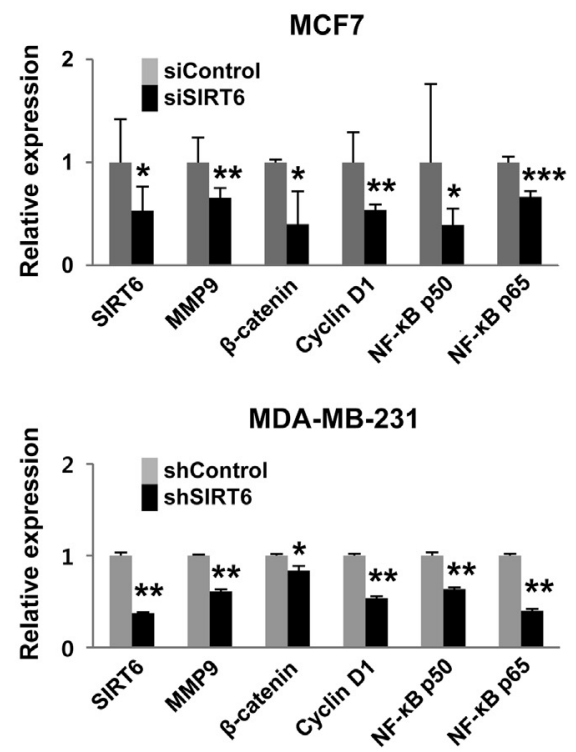

D

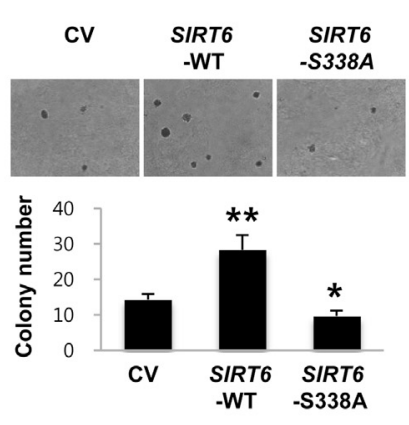

E

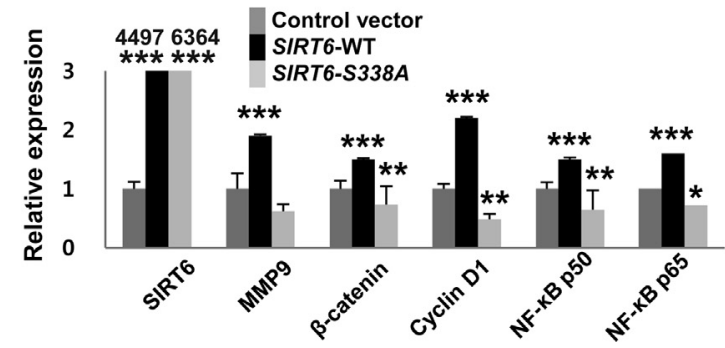

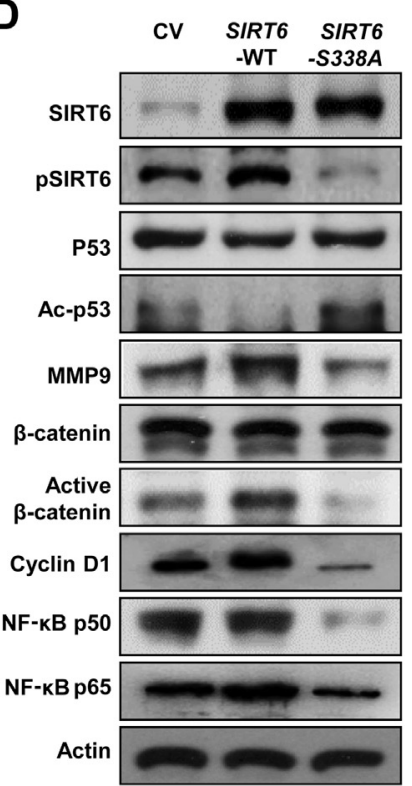

Figure 5 The expression and phosphorylation of SIRT6 is associated with the expression of the signaling molecules related to the proliferation of breast cancer cells. A and B: The protein and mRNA levels of MMP9, $\beta$-catenin, cyclin D1, and NF- $\mathrm{B} B$ decreased with knockdown of SIRT6 by siRNA or shRNA for SIRT6 in both MCF7 and MDA-MB-231 cells. C: Overexpression of SIRT6 induced by transfection of WT SIRT6 (SIRT6-WT; pFLAG2_SIRT6) increased proliferation of MCF7 cells. However, the proliferation of MCF7 cells decreased with the transfection of mutant SIRT6 (pSIRT6-S338A; pFLAG2_SIRT6_S338A). D: The transfection of WT SIRT6 increased expression of SIRT6, pSIRT6, MMP9, active $\beta$-catenin, cyclin D1, and NF- $\kappa$ B. The transfection of the SIRT6-S338A mutant decreased the level of pSIRT6 and the protein expression of MMP9, $\beta$-catenin, cyclin D1, and NF-KB. E: The transfection of WT SIRT6 increased expression of SIRT6, MMP9, $\beta$-catenin, cyclin D1, and NF- $\kappa$ B. In contrast, mutation of SIRT6 at the Ser338 phosphorylation site decreased the mRNA expression of $\beta$-catenin, cyclin D1, and NF- $\mathrm{BB} .{ }^{*} P<0.05,{ }^{*} P<0.01$, and ${ }^{* * *} P<0.001$. Ac-p53, acetylated p53; CV, control vector; MMP9, matrix metallopeptidase 9; $p$, phosphorylated; SIRT6, sirtuin 6; WT, wild-type.

Moreover, similar to the results found with a knockdown of SIRT6, transfection with a SIRT6-S338A mutant decreased the expression of mRNA and protein of MMP9, $\beta$-catenin, cyclin D1, and NF- $\kappa \mathrm{B}$ (Figure 5, D and E). The protein level of p53 was not changed, and acetylation of p53 was slightly increased with transfection of mutant SIRT6 (Figure 5D). The transfection of WT SIRT6 increased the mRNA of
SIRT6, MMP9, $\beta$-catenin, cyclin D1, and NF-кB (Figure 5E). These findings suggest that phosphorylation of SIRT6 is closely associated with the function of SIRT6 in the proliferation of MCF7 BCA cells.

Because SIRT6 was involved in the regulation of cell cycle and associated with both $\beta$-catenin and cyclin D1 expression, we further investigated the relationship between 
SIRT6 and $\beta$-catenin signaling. As expected, knockdown of $\beta$-catenin inhibited proliferation of both MCF7 and MDAMB-231 cell lines. In addition, overexpression of SIRT6 by transfection of SIRT6-WT increased proliferation of BCA cells, which was attenuated with a knockdown of $\beta$-catenin via shRNA for $\beta$-catenin (Figure 6A). The expression of protein of MMP9, cyclin D1, and NF- $\kappa$ B decreased with knockdown of $\beta$-catenin in both MCF7 and MDA-MB-231 cell lines. Overexpression of SIRT6 by transfection of SIRT6-WT increased protein levels of $\beta$-catenin, active $\beta$-catenin, MMP9, cyclin D1, and NF- $\kappa \mathrm{B}$, which decreased to the level of control cells with the cotransfection of shRNA for $\beta$-catenin (Figure 6B). Moreover, SIRT6 bound to $\beta$-catenin (Figure $6 \mathrm{C}$ ) and SIRT6 expression was associated with the localization of $\beta$-catenin in MCF7 cells (Figure 6, C-E). Western blot analysis with subcellular protein fractionation showed that nuclear and cytoplasmic expression of $\beta$-catenin decreased with knockdown of SIRT6 (Figure 6D); this was confirmed by confocal microscopic analysis (Figure 6E). In control MCF7 cells, the expression of $\beta$-catenin and active $\beta$-catenin was seen in both cell membranes and nuclei. However, when we induced a knockdown of SIRT6, $\beta$-catenin was expressed only in the cell membrane but not in the nuclei. The expression of active $\beta$-catenin also decreased with knockdown of SIRT6 (Figure 6E).

\section{Discussion}

In this study, we demonstrate that the expression of CSNK2A1 is closely associated with SIRT6/pSIRT6 expression and that the expression of both CSNK2A1 and SIRT6 were independent poor prognostic indicators for BCA patients. In agreement with our results, the expression of CSNK2A1 was suggested as an indicator of poor prognosis of BCA, ${ }^{9}$ gastric carcinoma, ${ }^{4}$ colon cancer, ${ }^{6,17}$ and prostate cancer. ${ }^{7}$ Thus, controlling the expression of CSNK2A1 might be a strategy in the treatment of the unfavorable subgroup of cancer patients expressing CSNK2A1. Recently, CSNK2 inhibitors have been under consideration as new chemotherapeutic agents. CX4945, a CSNK2 inhibitor, was shown to suppress cell migrationrelated phosphatidylinositol 3-kinase (PI3K)/Akt and mitogen-activated protein kinase (MAPK) pathways in lung cancer cells ${ }^{44}$ and to inhibit androgen receptordependent transcription in prostate cancer cells. ${ }^{45}$ Emodin was also shown to have anticancer activity. ${ }^{4,46}$ Our results have also shown that the proliferation of two BCA cell lines was inhibited via treatment of CX4945 and emodin as well as a siRNA-mediated knockdown of CSNK2A1. In addition, knockdown of CSNK2A1 induced apoptosis in MCF7 cells, induced expression of FOXO3 and PARP1, and inhibited migration and invasion of two BCA cell lines. Therefore, our results suggest that CSNK2A1 might be a potential therapeutic target for BCA patients with poor prognoses and who have tumors expressing CSNK2A1.

SIRTs have diverse roles in the regulation of intracellular mechanisms, and their roles have been reported according to the subtype of SIRTs, from SIRT1 to SIRT7. In our study, the expression of SIRT6 and pSIRT6 was associated with advanced clinicopathologic factors of BCAs, especially with the presence of distant metastatic relapse of BCA, and predicted shorter survival of BCA patients. In addition, knockdown of SIRT6 inhibited migration and invasion of BCA cells. The decreased invasive activity with a knockdown of SIRT6 was associated with decreased expression of MMP9. When considering the role of MMPs, especially MMP9, in the invasion and metastatic of cancer cells, ${ }^{47}$ our result suggests that SIRT6 is involved in the regulation of tumor invasiveness by regulating MMP9, which consequently, might influence the prognosis of BCA patients. In agreement with our results, SIRT6 promoted migration of pancreatic cancer cells by activating $\mathrm{Ca}^{2+}$ channels. ${ }^{28}$ In our study, knockdown of SIRT6 inhibited proliferation and proliferation-related signaling, and overexpression of WT SIRT6 increased proliferation of BCA cells. Similarly, SIRT6 increased the proliferation and survival of squamous cell carcinoma cells by activating cyclooxygenase- $2 .{ }^{27}$ Moreover, SIRT6 was involved in the survival and chemoresistance of BCA cells, and nuclear expression of SIRT6 predict poor survival of BCA patients. ${ }^{21}$ Therefore, these findings suggest that suppression of SIRT6 might have therapeutic potential for the treatment of BCA. In agreement with our results, nuclear expression of SIRT6 predicted shorter survival of BCA patients by multivariate analysis. However, cytoplasmic expression of SIRT6 was associated with favorable survival of BCA patients by univariate analysis. ${ }^{21}$ In addition, in contrast with our results, knockdown of SIRT6 increased the proliferation of MDA-MB-231 BCA cells, and expression of SIRT6 was associated with favorable prognosis of BCA patients. ${ }^{26}$ In other reports, SIRT6 was suggested to be a tumor suppressor by regulating aerobic glycolysis ${ }^{48}$ and the extracellular signal-related kinase $1 / 2$ pathway. ${ }^{24}$ Gene expression of SIRT6 was down-regulated in pancreatic and colonic cancer compared with normal counterpart tissue, and elevated expression of SIRT6 predicted longer disease-free survival in lymph node-positive colorectal cancers but not in node-negative colorectal cancers. $^{48}$ Nevertheless, as we have shown in Supplemental Figure S1, the public data from the Oncomine database showed that higher expression of SIRT6 mRNA was found in invasive BCAs than in normal breast tissue. Although it is not significant, the public data from the cBioPortal database showed that the median survival of BCA patients with genetic alteration of SIRT6 (amplification, deep deletion, truncated mutation, or missense mutation) is relatively shorter than the median survival of BCA patients with normal SIRT6 (Supplemental Figure S1B). ${ }^{49,50}$ In addition, our data have shown that 
A

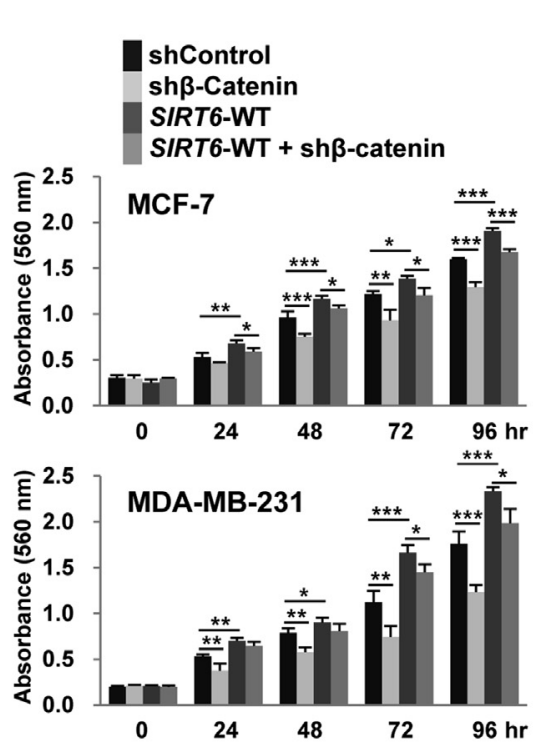

C

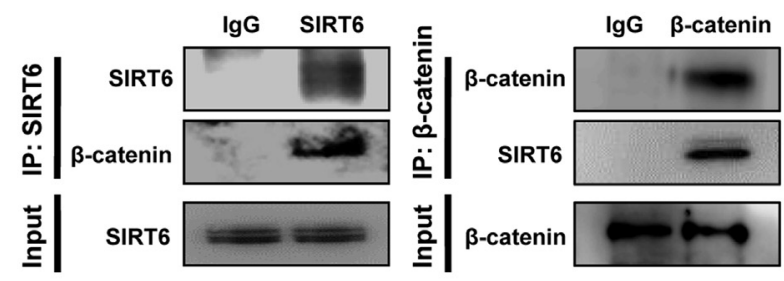

E

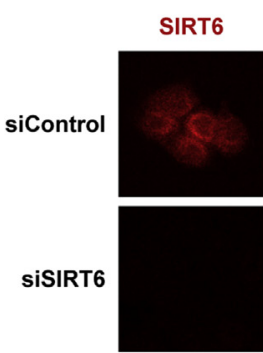

B

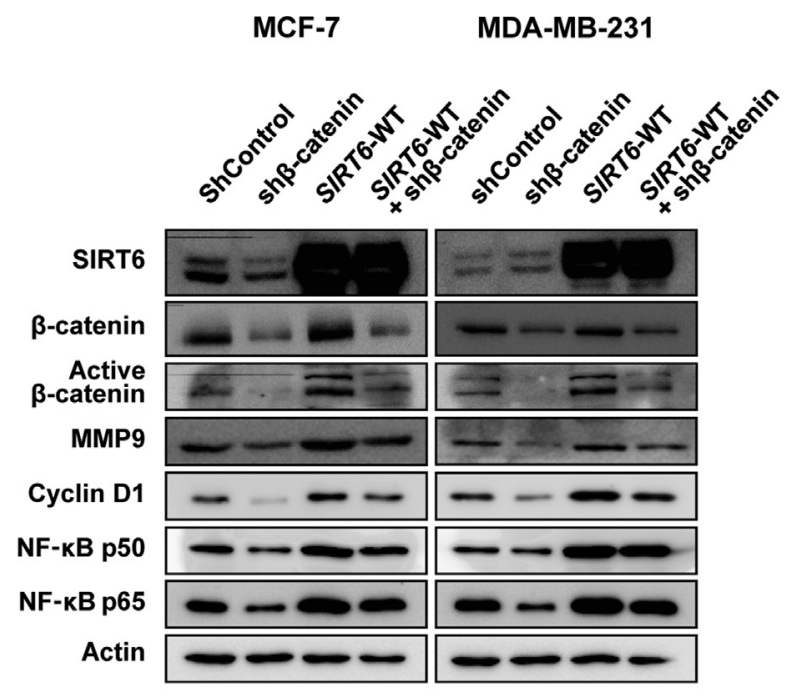

D

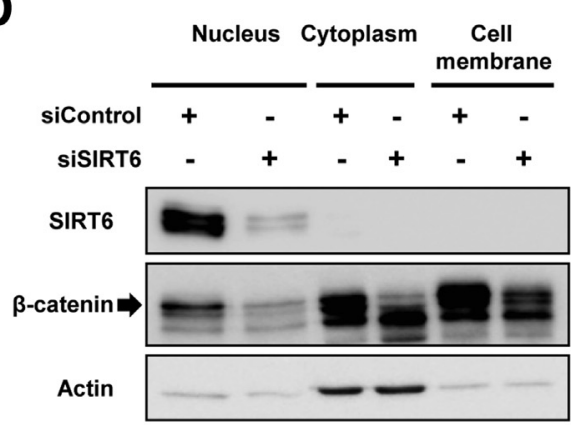

Figure 6 SIRT6 is involved the regulation of $\beta$-catenin expression. A: The knockdown of $\beta$-catenin inhibited proliferation of both MCF7 and MDA-MB-231 cell lines. Overexpression of SIRT6 by transfection of SIRT6-WT increased proliferation of both MCF7 and MDA-MB-231 cell lines. Increased proliferation of breast cancer cells by induction of SIRT6 overexpression was attenuated with knockdown of $\beta$-catenin with shRNA for $\beta$-catenin. B: The expression of protein of MMP9, cyclin D1, and NF- KB decreased with knockdown of $\beta$-catenin in both MCF7 and MDA-MB-231 cell lines. Overexpression of SIRT6 by transfection of SIRT6-WT increased protein levels of $\beta$-catenin, active $\beta$-catenin, MMP9, cyclin D1, and NF- $\kappa B$, which could be attenuated with cotransfection of shRNA for $\beta$-catenin. C: $\beta$-catenin was detected in samples via immunoprecipitation for SIRT6 and vice versa. D: Western blot analysis with subcellular protein fractionation indicated decreased nuclear and cytoplasmic expression of $\beta$-catenin in MCF7 cells after knockdown of SIRT6. E: Confocal microscopic images after immunofluorescence staining for SIRT6 and $\beta$-catenin show that $\beta$-catenin is expressed in both the cell membrane and nuclei of control MCF7 cells. However, $\beta$-catenin was expressed only in the cell membrane of MCF7 cells subjected to a knockdown of SIRT6. The images after co-immunofluorescence staining for SIRT6 and active $\beta$-catenin also demonstrated decreased nuclear expression of active $\beta$-catenin in MCF7 cells with a knockdown of SIRT6 compared with control MCF7 cells. ${ }^{*} P<0.05,{ }^{*} P<0.01$, and ${ }^{* *} P<0.001$. IP, immunoprecipitation; MMP9, matrix metallopeptidase 9; sh, short hairpin; si, small interfering; SIRT6, sirtuin 6; WT, wild-type.

the signaling molecules involved in the invasiveness and proliferation of BCA cells were down-regulated with a knockdown of SIRT6. Therefore, there is a possibility that the role of SIRT6 in tumorigenesis might differ according to cell type and/or tumor stage. Similarly, a controversial role of SIRTs in tumorigenesis has been reported for SIRT1 and SIRT3. ${ }^{18-20}$ Thus, further study is needed to explore the exact role of SIRT6 in tumorigenesis. 
A particularly interesting finding of our study is that CSNK2A1 was found to be involved in the phosphorylation of SIRT6 at Ser338. In addition to a role in phosphorylating SIRT1, ${ }^{13,30}$ CSNK2A1 was involved in the phosphorylation of SIRT6 on Ser338. pSIRT6 decreased with a knockdown of CSNK2A1. Moreover, we have shown evidence that CSNK2A1 binds to SIRT6 and causes its phosphorylation via immunofluorescence staining, co-IP of CSNK2A1 and SIRT6, a GST pull-down assay, an in vitro kinase assay, and transfection of mutant CSNK2A1. As we have shown in an in vitro kinase assay in Figure 3G, CSNK2A1 phosphorylates WT SIRT6, SIRT6-T294A mutant, SIRT6-S303A mutant, and SIRT6-S330A mutant proteins, but the SIRT6-S338A mutant protein was not phosphorylated by CSNK2A1. In addition, pSIRT6-Ser338 decreased with transfection of SIRT6-S338A, as shown in Figure 5D. These results show that CSNK2A1 is involved in the phosphorylation of SIRT6-Ser338. As we have shown that the phosphorylation of SIRT6-Ser338 is regulated by CSNK2A1, Thirumurthi et $\mathrm{al}^{26}$ presented that the phosphorylation of SIRT6 is controlled by AKT1 on Ser338 site. Therefore, it might be that the phosphorylation of SIRT6Ser338 is controlled by various kinases, including AKT1 and CSNK2A1.

When considering the accumulating evidence that CSNK2Al is a potent oncogene and the phosphorylation of target proteins of CSNK2A1, such as SIRT1 ${ }^{13,30}$ and DBC1/CCAR2, ${ }^{4}$ are tumorigenic, CSNK2A1-mediated phosphorylation of SIRT6 might also be tumorigenic. Especially, the kinase activity of CSNK2A1 augmented SIRT $1^{13,30}$ and the expression of SIRT1 were closely associated with human malignant tumors. ${ }^{13,20,35,51}$ Therefore, the oncogenic role of CSNK2A1 cannot be restricted to the regulation of the phosphorylation status of SIRT6. However, the role of SIRT6 in tumorigenesis is controversial. A recent report has shown that pSIRT6 is easily degraded by ubiquitination and that increased degradation of SIRT6 might be tumorigenic. ${ }^{26}$ In the study of Thirumurthi et al, ${ }^{26}$ transfection of a SIRT6-S338A mutant suppressed proliferation of cells, but a knockdown of SIRT6 increased the survival of MDA-MB-231 breast cancer cells. In addition, knockdown of SIRT6 was associated with resistance to trastuzumab, and it has been suggested that controlling SIRT6 expression might be a new strategy to overcome trastuzumab resistance. Although we did not evaluate the role of SIRT6 in chemoresistance, our clinical data suggest that SIRT6 expression might be associated with chemoresistance of BCA. When we separately analyzed in the subpopulation of $126 \mathrm{BCA}$ patients who received adjuvant chemotherapy, the expression of nuclear SIRT (OS, $P<0.001$; RFS, $P<0.001$ ), cytoplasmic SIRT6 (OS, $P<0.001$; RFS, $P<0.001$ ), nuclear pSIRT6 (OS, $P<0.001$; RFS, $P<0.001$ ), and cytoplasmic pSIRT6 (OS, $P=0.003$; RFS, $P=0.072$ ) were significantly associated with shorter survival of BCA patients (Supplemental Figure S2). In addition, as we have shown in Table 3, immunohistochemical expression of nuclear SIRT6 $(P=0.001)$, cytoplasmic SIRT6 $(P=0.004)$, nuclear pSIRT6 $(P<0.001)$, and cytoplasmic pSIRT6 $(P=0.001)$ were significantly associated with HER2 expression. Although it is not clear why pSIRT6 plays a more significant role in the survival of BCA patients when it is localized in the nucleus than when it is localized in the cytosol, it might be related with the major role of nuclear SIRT6 in the survival of BCA patients. There was significant association between the expression patterns of nuclear SIRT6, cytoplasmic SIRT6, nuclear pSIRT6, and cytoplasmic pSIRT6. In addition, as we have shown in Table 4, nuclear SIRT6 expression was an independent indicator of poor prognosis by the multivariate analysis model 1 performed with inclusion of the expression of nuclear SIRT6, cytoplasmic SIRT6, nuclear

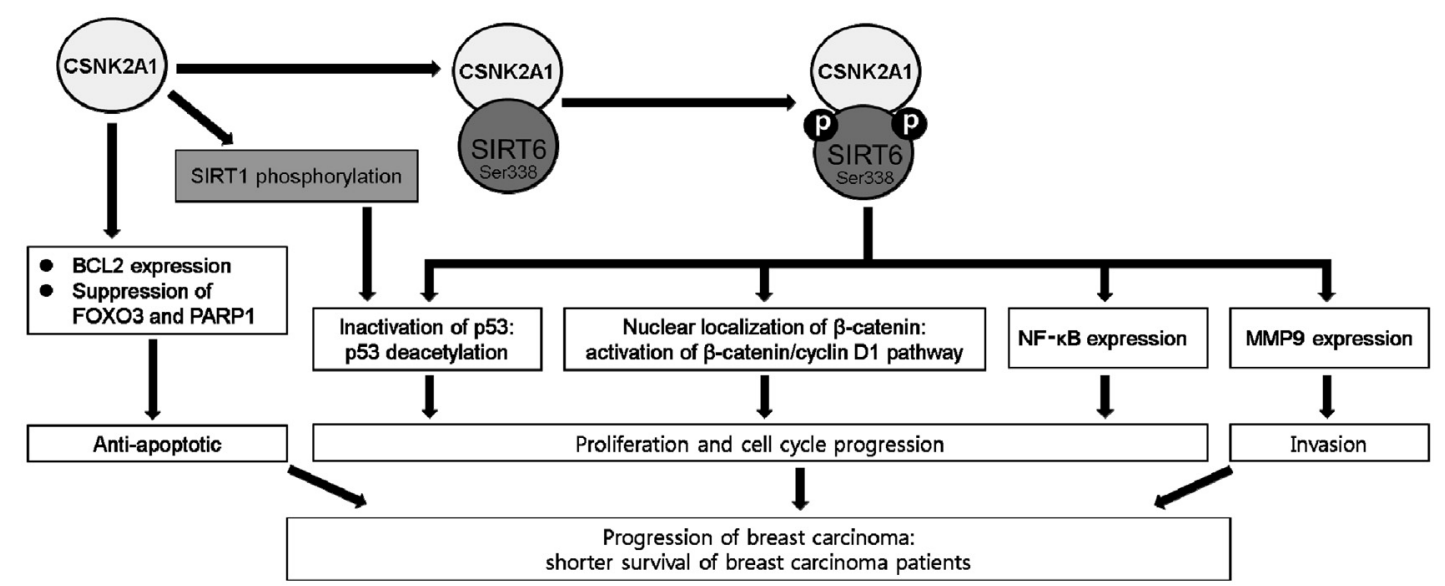

Figure 7 Schematic representation of the role of CSNK2A1 and SIRT6 in the progression of breast carcinoma. CSNK2A1 phosphorylates diverse target proteins, including SIRT1 and SIRT6. Especially, CSNK2A1 phosphorylates SIRT6 on Ser338. Expression of SIRT6 and its phosphorylation is involved in the progression of breast carcinoma via activation of the $\beta$-catenin/cyclin D1 pathway, expression of NF- $\kappa \mathrm{B}$ and MMP9, and deacetylation-mediated inactivation of p53. BCL2, B-cell lymphoma 2; CSNK2A1, protein kinase CK2 $\alpha 1$; F0X03, forkhead box 03; MMP9, matrix metallopeptidase 9; p, phosphorylated; PARP1, poly (ADP-ribose) polymerase 1; SIRT6, sirtuin 6. 
pSIRT6, and cytoplasmic pSIRT6. Moreover, when we evaluated the effect of SIRT6 expression and SIRT6 phosphorylation on tumor-related signaling, both knockdown of SIRT6 and mutation of SIRT6 at its Ser338 phosphorylation site inhibited the expression of the signals potentially important in tumorigenesis, such as MMP9- and $\beta$-catenin-related signaling. Therefore, there is speculation that nuclear pSIRT6 might affect the progression of BCA by involvement in the regulation of the expression of $\beta$-catenin, cyclin D1, and NF- $\kappa \mathrm{B}$. However, the precise mechanism of how Ser338 phosphorylation enhances the oncogenic activities of SIRT6 in BCA is not clear. Therefore, further study is needed to clarify the role of SIRT6 in BCA tumorigenesis and chemoresistance.

The Wnt/ $\beta$-catenin pathway is important in the proliferation and survival of cells. ${ }^{52-54}$ The critical event for the effects of $\beta$-catenin is its nuclear translocation from the cytosol and binding to $\mathrm{T}$ cell transcription factor to induce proliferation-related signaling. ${ }^{55}$ Although there are some exceptions, ${ }^{56}$ the nuclear localization of $\beta$-catenin is important for the progression of malignant tumors and it predicted poor survival. ${ }^{44}$ The results of this study suggest that the oncogenic role of SIRT6 is mediated by regulating $\beta$-catenin pathway-mediated proliferation. The knockdown of SIRT6 and a mutation at the phosphorylation site of SIRT6 decreased expression levels of protein of $\beta$-catenin, cyclin D1, and NF- $\kappa \mathrm{B}$. In addition, the increased proliferation of BCA cells and increased expression of the signaling molecules related to proliferation by inducing SIRT6 overexpression was attenuated by cotransfection of shRNA for $\beta$-catenin. Moreover, SIRT6 bound directly to $\beta$-catenin and knockdown of SIRT6 affected the nuclear localization of $\beta$-catenin. Therefore, our result suggests that SIRT6 might be involved in the proliferation of BCA cells by engaging $\beta$-catenin signaling. When considering the role of CSNK2A1 for the phosphorylation of various proteins, including SIRT6, it was expected that active (dephosphorylated) $\beta$-catenin increased with knockdown of CSNK2A1 or SIRT6; however, surprisingly, the expression of active $\beta$-catenin decreased with the knockdown of SIRT6. In addition, although, a recent report showed that CSNK2A1 is involved in the proliferation of glioblastoma by regulating the $\beta$-catenin pathway, ${ }^{57}$ the expression of $\beta$-catenin and active $\beta$-catenin were not changed with a knockdown of CSNK2Al in our study (Supplemental Figure S3). Therefore, further study is needed to explore the relationship between SIRT6 and $\beta$-catenin in tumorigenesis.

\section{Conclusions}

In conclusion, as we summarized in Figure 7, this study demonstrates that CSNK2A1 phosphorylates SIRT6 on Ser338 and the expression of SIRT6 and its phosphorylation is involved in the progression of BCA via activation of the $\beta$-catenin/cyclin D1 pathway, expression of NF- $\kappa \mathrm{B}$ and MMP9, and deacetylation-mediated inactivation of p53. Moreover, the expression of CSNK2A1 and SIRT6 was and independent indicator of poor prognosis of BCA patients. In addition, the blocking of CSNK2A1 and SIRT6 inhibited the proliferation and invasiveness of BCA cells. Therefore, this study suggests that the CSNK2A1-SIRT6 pathway might be a new therapeutic target for the treatment of BCA.

\section{Acknowledgments}

We thank Yeon Gyu Yu for providing the GST-fusion CSNK2A1 expression vectors, Laszlo Gyenis and Suk Woo Nam for providing the plasmids for wild-type CSNK2A1 and mutant CSNK2A1, and Dennis B. Leveson-Gower who provided medical writing services.

J.S.B., S.-H.P., U.J., S.J.N., J.R.K., K.S.K., S.H.J., H.S.P., B.-H.P., H.L., W.S.M., K.G.S., and K.Y.J. were responsible for study design and data interpretation. J.S.B., S.-H.P., U.J., K.M.K., S.J.N., H.J.P., K.S.K., S.H.J., H.S.P., B.-H.P., and K.Y.J. performed the experiments. J.S.B., S.-H.P., U.J., K.M.K., S.J.N., J.R.K., H.J.P., K.S.K., S.H.J., H.S.P., B.-H.P., H.L., W.S.M., K.G.S., and K.Y.J. analyzed the data. J.S.B., S.-H.P., U.J., K.M.K., S.J.N., J.R.K., H.J.P., K.S.K., H.S.P., B.-H.P., H.L., W.S.M., K.G.S., and K.Y.J. wrote the paper. All authors have critically read, edited, and approved the final version of the manuscript.

\section{Supplemental Data}

Supplemental material for this article can be found at http://dx.doi.org/10.1016/j.ajpath.2016.08.007.

\section{References}

1. Meggio F, Pinna LA: One-thousand-and-one substrates of protein kinase CK2? FASEB J 2003, 17:349-368

2. Canton DA, Litchfield DW: The shape of things to come: an emerging role for protein kinase CK2 in the regulation of cell morphology and the cytoskeleton. Cell Signal 2006, 18:267-275

3. Ruzzene M, Pinna LA: Addiction to protein kinase CK2: a common denominator of diverse cancer cells? Biochim Biophys Acta 2010, 1804:499-504

4. Bae JS, Park SH, Kim KM, Kwon KS, Kim CY, Lee HK, Park BH, Park HS, Lee H, Moon WS, Chung MJ, Sylvester KG, Jang KY: CK2alpha phosphorylates DBC1 and is involved in the progression of gastric carcinoma and predicts poor survival of gastric carcinoma patients. Int J Cancer 2015, 136:797-809

5. Stalter G, Siemer S, Becht E, Ziegler M, Remberger K, Issinger OG: Asymmetric expression of protein kinase CK2 subunits in human kidney tumors. Biochem Biophys Res Commun 1994, 202:141-147

6. Zou J, Luo H, Zeng Q, Dong Z, Wu D, Liu L: Protein kinase CK2alpha is overexpressed in colorectal cancer and modulates cell proliferation and invasion via regulating EMT-related genes. J Transl Med 2011, 9:97

7. Laramas M, Pasquier D, Filhol O, Ringeisen F, Descotes JL, Cochet C: Nuclear localization of protein kinase CK2 catalytic subunit (CK2alpha) is associated with poor prognostic factors in human prostate cancer. Eur J Cancer 2007, 43:928-934 
8. Landesman-Bollag E, Romieu-Mourez R, Song DH, Sonenshein GE, Cardiff RD, Seldin DC: Protein kinase CK2 in mammary gland tumorigenesis. Oncogene 2001, 20:3247-3257

9. Giusiano S, Cochet C, Filhol O, Duchemin-Pelletier E, Secq V, Bonnier P, Carcopino X, Boubli L, Birnbaum D, Garcia S, Iovanna J, Charpin C: Protein kinase CK2alpha subunit over-expression correlates with metastatic risk in breast carcinomas: quantitative immunohistochemistry in tissue microarrays. Eur J Cancer 2011, 47: 792-801

10. Bousset K, Oelgeschlager MH, Henriksson M, Schreek S, Burkhardt H, Litchfield DW, Luscher-Firzlaff JM, Luscher B: Regulation of transcription factors c-Myc, Max, and c-Myb by casein kinase II. Cell Mol Biol Res 1994, 40:501-511

11. Song DH, Dominguez I, Mizuno J, Kaut M, Mohr SC, Seldin DC: CK2 phosphorylation of the armadillo repeat region of beta-catenin potentiates Wnt signaling. J Biol Chem 2003, 278:24018-24025

12. Chantome A, Pance A, Gauthier N, Vandroux D, Chenu J, Solary E, Jeannin JF, Reveneau S: Casein kinase II-mediated phosphorylation of NF-kappaB p65 subunit enhances inducible nitric-oxide synthase gene transcription in vivo. J Biol Chem 2004, 279:23953-23960

13. Cha EJ, Noh SJ, Kwon KS, Kim CY, Park BH, Park HS, Lee H, Chung MJ, Kang MJ, Lee DG, Moon WS, Jang KY: Expression of DBC1 and SIRT1 is associated with poor prognosis of gastric carcinoma. Clin Cancer Res 2009, 15:4453-4459

14. Al-Khouri AM, Ma Y, Togo SH, Williams S, Mustelin T: Cooperative phosphorylation of the tumor suppressor phosphatase and tensin homologue (PTEN) by casein kinases and glycogen synthase kinase 3beta. J Biol Chem 2005, 280:35195-35202

15. Dixit D, Sharma V, Ghosh S, Mehta VS, Sen E: Inhibition of Casein kinase-2 induces p53-dependent cell cycle arrest and sensitizes glioblastoma cells to tumor necrosis factor (TNFalpha)-induced apoptosis through SIRT1 inhibition. Cell Death Dis 2012, 3:e271

16. Scaglioni PP, Yung TM, Cai LF, Erdjument-Bromage H, Kaufman AJ, Singh B, Teruya-Feldstein J, Tempst P, Pandolfi PP: A CK2-dependent mechanism for degradation of the PML tumor suppressor. Cell 2006, 126:269-283

17. Lin KY, Tai C, Hsu JC, Li CF, Fang CL, Lai HC, Hseu YC, Lin YF, Uen YH: Overexpression of nuclear protein kinase CK2 alpha catalytic subunit (CK2alpha) as a poor prognosticator in human colorectal cancer. PLoS One 2011, 6:e17193

18. Alhazzazi TY, Kamarajan P, Verdin E, Kapila YL: SIRT3 and cancer: tumor promoter or suppressor? Biochim Biophys Acta 2011, $1816: 80-88$

19. Deng CX: SIRT1, is it a tumor promoter or tumor suppressor? Int J Biol Sci 2009, 5:147-152

20. Jang KY, Noh SJ, Lehwald N, Tao GZ, Bellovin DI, Park HS, Moon WS, Felsher DW, Sylvester KG: SIRT1 and c-Myc promote liver tumor cell survival and predict poor survival of human hepatocellular carcinomas. PLoS One 2012, 7:e45119

21. Khongkow M, Olmos Y, Gong C, Gomes AR, Monteiro LJ, Yague E, Cavaco TB, Khongkow P, Man EP, Laohasinnarong S, Koo CY, Harada-Shoji N, Tsang JW, Coombes RC, Schwer B, Khoo US, Lam EW: SIRT6 modulates paclitaxel and epirubicin resistance and survival in breast cancer. Carcinogenesis 2013, 34:1476-1486

22. Kim HS, Xiao C, Wang RH, Lahusen T, Xu X, Vassilopoulos A, Vazquez-Ortiz G, Jeong WI, Park O, Ki SH, Gao B, Deng CX: Hepatic-specific disruption of SIRT6 in mice results in fatty liver formation due to enhanced glycolysis and triglyceride synthesis. Cell Metab 2010, 12:224-236

23. Mostoslavsky R, Chua KF, Lombard DB, Pang WW, Fischer MR, Gellon L, Liu P, Mostoslavsky G, Franco S, Murphy MM, Mills KD Patel P, Hsu JT, Hong AL, Ford E, Cheng HL, Kennedy C, Nunez N, Bronson R, Frendewey D, Auerbach W, Valenzuela D, Karow M, Hottiger MO, Hursting S, Barrett JC, Guarente L, Mulligan R, Demple B, Yancopoulos GD, Alt FW: Genomic instability and aginglike phenotype in the absence of mammalian SIRT6. Cell 2006, 124: 315-329
24. Zhang ZG, Qin CY: Sirt6 suppresses hepatocellular carcinoma cell growth via inhibiting the extracellular signal-regulated kinase signaling pathway. Mol Med Rep 2014, 9:882-888

25. Marquardt JU, Fischer K, Baus K, Kashyap A, Ma S, Krupp M, Linke M, Teufel A, Zechner U, Strand D, Thorgeirsson SS, Galle PR, Strand S: Sirtuin-6-dependent genetic and epigenetic alterations are associated with poor clinical outcome in hepatocellular carcinoma patients. Hepatology 2013, 58:1054-1064

26. Thirumurthi U, Shen J, Xia W, LaBaff AM, Wei Y, Li CW, Chang WC, Chen CH, Lin HK, Yu D, Hung MC: MDM2-mediated degradation of SIRT6 phosphorylated by AKT1 promotes tumorigenesis and trastuzumab resistance in breast cancer. Sci Signal 2014, 7:ra71

27. Ming M, Han W, Zhao B, Sundaresan NR, Deng CX, Gupta MP, He YY: SIRT6 promotes COX-2 expression and acts as an oncogene in skin cancer. Cancer Res 2014, 74:5925-5933

28. Bauer I, Grozio A, Lasiglie D, Basile G, Sturla L, Magnone M, Sociali G, Soncini D, Caffa I, Poggi A, Zoppoli G, Cea M, Feldmann G, Mostoslavsky R, Ballestrero A, Patrone F, Bruzzone S, Nencioni A: The NAD+-dependent histone deacetylase SIRT6 promotes cytokine production and migration in pancreatic cancer cells by regulating Ca2 + responses. J Biol Chem 2012, 287: 40924-40937

29. Feng XX, Luo J, Liu M, Yan W, Zhou ZZ, Xia YJ, Tu W, Li PY, Feng ZH, Tian DA: Sirtuin 6 promotes transforming growth factorbeta $/ / \mathrm{H} 2 \mathrm{O} 2 / \mathrm{HOCl}$-mediated enhancement of hepatocellular carcinoma cell tumorigenicity by suppressing cellular senescence. Cancer Sci 2015, 106:559-566

30. Zschoernig B, Mahlknecht U: Carboxy-terminal phosphorylation of SIRT1 by protein kinase CK2. Biochem Biophys Res Commun 2009, 381:372-377

31. Miteva YV, Cristea IM: A proteomic perspective of Sirtuin 6 (SIRT6) phosphorylation and interactions and their dependence on its catalytic activity. Mol Cell Proteomics 2014, 13:168-183

32. Lakhani SR, Ellis IO, Schnitt SJ, Tan PH, van de Vijver MJ: WHO Classification of Tumours of the Breast. Lyon, France, IARC, 2012

33. Edge SB, Byrd DR, Compton CC, Fritz AG, Greene FL, Trotti A AJCC Cancer Staging Manual. ed 7. New York, Springer, 2009

34. Allred D, Harvey JM, Berardo M, Clark GM: Prognostic and predictive factors in breast cancer by immunohistochemical analysis. Mod Pathol 1998, 11:155-168

35. Kim JR, Moon YJ, Kwon KS, Bae JS, Wagle S, Yu TK, Kim KM, Park HS, Lee JH, Moon WS, Lee H, Chung MJ, Jang KY: Expression of SIRT1 and DBC1 is associated with poor prognosis of soft tissue sarcomas. PLoS One 2013, 8:e74738

36. Noh SJ, Kang MJ, Kim KM, Bae JS, Park HS, Moon WS, Chung MJ, Lee H, Lee DG, Jang KY: Acetylation status of P53 and the expression of DBC1, SIRT1, and androgen receptor are associated with survival in clear cell renal cell carcinoma patients. Pathology 2013, 45:574-580

37. Wagle S, Park SH, Kim KM, Moon YJ, Bae JS, Kwon KS, Park HS, Lee H, Moon WS, Kim JR, Jang KY: DBC1/CCAR2 is involved in the stabilization of androgen receptor and the progression of osteosarcoma. Sci Rep 2015, 5:13144

38. Siddiqui-Jain A, Drygin D, Streiner N, Chua P, Pierre F, O'Brien SE, Bliesath J, Omori M, Huser N, Ho C, Proffitt C, Schwaebe MK, Ryckman DM, Rice WG, Anderes K: CX-4945, an orally bioavailable selective inhibitor of protein kinase CK2, inhibits prosurvival and angiogenic signaling and exhibits antitumor efficacy. Cancer Res 2010, 70:10288-10298

39. Li XX, Dong Y, Wang W, Wang HL, Chen YY, Shi GY, Yi J, Wang J: Emodin as an effective agent in targeting cancer stem-like side population cells of gallbladder carcinoma. Stem Cells Dev 2013, 22:554-566

40. Huang Z, Chen G, Shi P: Emodin-induced apoptosis in human breast cancer BCap-37 cells through the mitochondrial signaling pathway. Arch Pharm Res 2008, 31:742-748 
41. Kim YK, Lee KJ, Jeon H, Yu YG: Protein kinase CK2 is inhibited by human nucleolar phosphoprotein p140 in an inositol hexakisphosphate-dependent manner. J Biol Chem 2006, 281: 36752-36757

42. Rhodes DR, Yu J, Shanker K, Deshpande N, Varambally R, Ghosh D, Barrette T, Pandey A, Chinnaiyan AM: ONCOMINE: a cancer microarray database and integrated data-mining platform. Neoplasia 2004, 6:1-6

43. Kim HS, Chang YG, Bae HJ, Eun JW, Shen Q, Park SJ, Shin WC, Lee EK, Park S, Ahn YM, Park WS, Lee JY, Nam SW: Oncogenic potential of CK2alpha and its regulatory role in EGF-induced HDAC2 expression in human liver cancer. FEBS J 2014, 281: $851-861$

44. Ku MJ, Park JW, Ryu BJ, Son YJ, Kim SH, Lee SY: CK2 inhibitor CX4945 induces sequential inactivation of proteins in the signaling pathways related with cell migration and suppresses metastasis of A549 human lung cancer cells. Bioorg Med Chem Lett 2013, 23: $5609-5613$

45. Ryu BJ, Baek SH, Kim J, Bae SJ, Chang SY, Heo JN, Lee H, Lee SY, Kim SH: Anti-androgen receptor activity of apoptotic CK2 inhibitor CX4945 in human prostate cancer LNCap cells. Bioorg Med Chem Lett 2012, 22:5470-5474

46. Santarpia L, Iwamoto T, Di Leo A, Hayashi N, Bottai G, Stampfer M, André F, Turner NC, Symmans WF, Hortobágyi GN, Pusztai L, Bianchini G: DNA repair gene patterns as prognostic and predictive factors in molecular breast cancer subtypes. Oncologist 2013, 18: 1063-1073

47. Deryugina EI, Quigley JP: Matrix metalloproteinases and tumor metastasis. Cancer Metastasis Rev 2006, 25:9-34

48. Sebastian C, Zwaans BM, Silberman DM, Gymrek M, Goren A, Zhong L, Ram O, Truelove J, Guimaraes AR, Toiber D, Cosentino C, Greenson JK, MacDonald AI, McGlynn L, Maxwell F, Edwards J, Giacosa S, Guccione E, Weissleder R, Bernstein BE, Regev A, Shiels PG, Lombard DB, Mostoslavsky R: The histone deacetylase SIRT6 is a tumor suppressor that controls cancer metabolism. Cell 2012, 151:1185-1199
49. Cerami E, Gao J, Dogrusoz U, Gross BE, Sumer SO, Aksoy BA, Jacobsen A, Byrne CJ, Heuer ML, Larsson E, Antipin Y, Reva B, Goldberg AP, Sander C, Schultz N: The cBio cancer genomics portal: an open platform for exploring multidimensional cancer genomics data. Cancer Discov 2012, 2:401-404

50. Gao J, Aksoy BA, Dogrusoz U, Dresdner G, Gross B, Sumer SO, Sun Y, Jacobsen A, Sinha R, Larsson E, Cerami E, Sander C, Schultz N: Integrative analysis of complex cancer genomics and clinical profiles using the cBioPortal. Sci Signal 2013, 6:p11

51. Jang KY, Hwang SH, Kwon KS, Kim KR, Choi HN, Lee NR, Kwak JY, Park BH, Park HS, Chung MJ, Kang MJ, Lee DG, Kim HS, Shim H, Moon WS: SIRT1 expression is associated with poor prognosis of diffuse large B-cell lymphoma. Am J Surg Pathol 2008, 32:1523-1531

52. Lehwald N, Tao GZ, Jang KY, Sorkin M, Knoefel WT, Sylvester KG: Wnt-beta-catenin signaling protects against hepatic ischemia and reperfusion injury in mice. Gastroenterology 2011, 141: 707-718

53. Tao GZ, Lehwald N, Jang KY, Baek J, Xu B, Omary MB, Sylvester KG: Wnt/beta-catenin signaling protects mouse liver against oxidative stress-induced apoptosis through the inhibition of forkhead transcription factor FoxO3. J Biol Chem 2013, 288: $17214-17224$

54. Wong CM, Fan ST, Ng IO: beta-Catenin mutation and overexpression in hepatocellular carcinoma: clinicopathologic and prognostic significance. Cancer 2001, 92:136-145

55. Nelson WJ, Nusse R: Convergence of Wnt, beta-catenin, and cadherin pathways. Science 2004, 303:1483-1487

56. Jang KY, Kim YN, Bae JS, Chung MJ, Moon WS, Kang MJ, Lee DG, Park HS: Expression of cyclin D1 is associated with betacatenin expression and correlates with good prognosis in colorectal adenocarcinoma. Transl Oncol 2012, 5:370-378

57. Nitta RT, Gholamin S, Feroze AH, Agarwal M, Cheshier SH, Mitra SS, Li G: Casein kinase 2alpha regulates glioblastoma brain tumor-initiating cell growth through the beta-catenin pathway. Oncogene 2015, 34:3688-3699 